\title{
THE RIEMANN SPHERE OF A COMMUTATIVE BANACH ALGEBRA $\left({ }^{1}\right)$
}

\author{
BY \\ BARNETT W. GLICKFELD
}

Introduction. The Riemann sphere, or extended complex plane $C_{\infty}$, has long played an important role in classical function theory. Therefore in abstract function theory, where the range or both the domain and range of an analytic function lie in a Banach space or algebra $A$, it is natural to pose the problem: how should $A$ be extended to a new structure $A_{\infty}$ which plays the role of the Riemann sphere? Here we give the solution to this problem, valid when $A$ is a complex commutative Banach algebra with identity. $A_{\infty}$ is provided with quasi-algebraic, topological, and analytic structure.

In $\S 1$, the quasi-algebraic structure of $A_{\infty}$ is studied, with the exposition being given for the surprisingly abstract context of a commutative ring with identity. We say quasi-algebraic rather than algebraic because elements of $A_{\infty}$ may only sometimes be added and sometimes multiplied. $A_{\infty}$ may be regarded as the set obtained by adjoining to $A$ all formal quotients $a / b$ of elements of $A$, where $b$ is singular and both $a$ and $b$ lie in no proper ideal of $A$. The $2 \times 2$ matrices with coefficients in $A$ and invertible determinant induce the fractional linear group of bijections of $A_{\infty}$. Ring homomorphisms induce Riemann sphere homomorphisms and fractional linear group homomorphisms which are related; this lends a functorial tinge to the subject.

In $\S 2$, we equip $A_{\infty}$ (where $A$ is now required to be a commutative complex $B$-algebra with unit) with the unique topology so that $A$ is an open subspace and each fractional linear transformation is a homeomorphism. Locally compact Riemann spheres occur iff $A$ is finite dimensional, and compact Riemann spheres are even rarer. The lifting of algebra homomorphisms to Riemann sphere homomorphisms enables the extension of the Gelfand representation of $A$ to $A_{\infty}$, as well as the definition of the spectrum of an element of $A_{\infty}$.

The Riemann sphere construction enables us to replace one disconnectedness phenomenon by another. The mapping $a \rightarrow a^{-1}$ is usually regarded as being defined on the (perhaps not connected) set $I$ of invertible elements of $A$. However, here we regard $a \rightarrow a^{-1}$ as a mapping of all of $A_{\infty}$ onto itself, and clearly $I$ is contained in the component of $A_{\infty}$ which contains $A$. Unfortunately Example 2.6.5 shows that $A_{\infty}$ itself need not be connected. A related phenomenon is that the spectrum of an element of $A_{\infty}$ may be the whole extended plane; whenever $A_{\infty}$ is disconnected there are such elements, but not conversely.

In $\S 3$, we give $A_{\infty}$ an analytic structure, in which each fractional transformation

Received by the editors October 8, 1965 and, in revised form, June 7, 1967.

( ${ }^{1}$ Supported by a U.S. Army (Durham) contract. 
is an analytic automorphism, via the Lorch analytic function theory. We assume only the most basic results of this theory, all of which may be found in Lorch's original paper [6]. Excellent discussions illuminating the nature of this theory and its relation to the other abstract function theories are to be found in [7] and [8]. A fairly complete account of the various analytic function theories in $B$-spaces and $B$-algebras is available in Hille and Phillips [5].

The central theorem of $\$ 3$ is the generalization of the classical theorem that each entire function which approaches infinity at infinity is a complex polynomial. The appropriate formulation of this generalization requires that special attention be paid to the radical of $A$, as well as to the nontrivial idempotents of $A$. (If $A$ is semisimple, and has no nontrivial idempotents, then each analytic function on $A$, with a limit at infinity in an appropriate sense, is a polynomial on $A$ with invertible leading coefficient.) As a corollary, the group of analytic automorphisms of the connected component of $A_{\infty}$ which contains $A$ is shown, via the classical monodromy theorem, to be the fractional linear group.

REMARK. Although this paper is oriented towards abstract function theory and spectral theory, the Riemann sphere construction is of topological-algebraic interest, since it associates to each commutative (real or complex, although we only consider complex scalars in the paper proper) $B$-algebra with identity $A$, a topological space $A_{\infty}$ which is a topological-algebraic invariant of $A$, but not a topological invariant. It is interesting to consider the three real Banach algebras obtained by taking the plane $R^{2}$, with pointwise linear operations, and the multiplications and norms given by

(1) $(s, t)(u, v)=(s u, t v), \quad\|(s, t)\|=\max (|s|,|t|)$,

(2) $(s, t)(u, v)=(s u-t v, s v+u t), \quad\|(s, t)\|=\left(|s|^{2}+|t|^{2}\right)^{1 / 2}$,

(3) $(s, t)(u, v)=(s u, s v+u t), \quad\|(s, t)\|=|s|+|t|$.

In (1) the Riemann sphere is the surface of a torus, in (2) it is the classical Riemann sphere $C_{\infty}$, and in (3) it is the surface of an open cylinder.

With the exception of 3.4, the results of this paper are contained in the author's Columbia University dissertation [4], which also contains other results bearing on the Lorch theory in general, and on this paper in particular. In a forthcoming paper, we will discuss the applications of the Riemann sphere to operational calculus, with particular reference to the work of Taylor on unbounded closed operators [11].

We should note that de Bruijn [2] has sketched a different definition of Riemann sphere, valid for a not necessarily commutative Banach algebra $A$. In the commutative case, his sphere is the smallest subset of $A_{\infty}$ which contains $A$ and is invariant under the complex fractional linear transformations. This notion is not strong enough to permit the definition of such quotients as $\sin a / \cos a$ for singular $\cos a$. De Bruijn's treatment involves neither the ideals of $A$ nor the algebraic structure of $A_{\infty}$, both of which play an important role here. In [2] the concept of the Riemann sphere as an "analytic $A$-manifold" is introduced. 
I am happy to acknowledge my many invaluable discussions with $\mathrm{E}$. R. Lorch about analytic function theory in Banach algebras, during which he pointed out the need for a theory of fractional linear transformations, and suggested that the next stage in the evolution of function theory in a Banach algebra should be the mixture of classical function-theoretic notions with the ideal theory of the algebra. I would also like to acknowledge several stimulating conversations with $\mathrm{R}$. Bott, and the help of $\mathrm{H}$. Appelgate with Example 2.6.5. The referee has considerably simpiined the proof of 3.1.1.

Notation and terminology. Let $S, S_{1}$ and $S_{2}$ be sets.

1. The identity map of $S$ onto itself is denoted by $1_{S}$ or $1(S)$.

2. If $f: S_{1} \rightarrow S_{2}$ and $S \subseteq S_{1}, f \mid S=$ the restriction of $f$ to $S$.

3. If $f: S \rightarrow S_{1}$ and $g: S_{1} \rightarrow S_{2}$, then $g \circ f$ denotes the composition of $f$ and $g$.

Let $Y$ be a topological space.

4. $D$ is a domain in $Y$ iff $D$ is a region in $Y$ iff $D$ is an open connected subset of $Y$.

5. $C(Y)=$ the Banach algebra of all continuous bounded complex-valued functions on $Y$ with pointwise algebraic operations and the sup norm.

6. $C=$ the complex number field.

Let $A$ be a commutative complex Banach algebra with identity.

7. $A$ is irreducible iff the only idempotents in $A$ are 0 and 1.

8. $\mathscr{M}=$ the maximal ideal space of $A$. As usual, we identify the maximal ideals $M$ of $A$ with the associated complex algebra homomorphisms $F: A \rightarrow C$.

9. $\wedge$ denotes the Gelfand representation of $A$ into $C(\mathscr{M})$, while $\hat{A}$ denotes the image of $\bumpeq$.

10. As is usual, we identify the complex number 1 with the identity element of $A$. Thus $C$ is considered to be a subset of $A$.

11. $z$ will be used to denote complex numbers and complex variables, while $a$ will denote elements of $A$ and $A$-variables.

12. If $a_{0} \in A$ and $R$ is a nonnegative extended real number, $B\left(a_{0} ; R\right)$ denotes the open norm ball of radius $R$ about $a_{0}$, while $\bar{B}\left(a_{0} ; R\right)$ denotes the closed norm ball of radius $R$ about $a_{0}$.

13. If $z_{0} \in C$ and $R$ is a nonnegative extended real number, $K\left(z_{0} ; R\right)$ denotes the open disc in the complex plane of radius $R$ about $z_{0}$, while $\bar{K}\left(z_{0} ; R\right)$ denotes the closed disc in the complex plane of radius $R$ about $z_{0}$.

14. If $A$-domains and $C$-domains are under consideration at the same time, the $C$-domains will be called complex domains, while the $A$-domains will simply be called domains. Similar conventions hold with respect to $C$-holomorphic functions and $A$-holomorphic functions, etc.

15. In general, the terminology used for classical function theory will be that found in Saks and Zygmund [10]. The terminology used for abstract function theory will parallel that used for classical function theory, except in the introduction. 
1. The Riemann sphere of a commutative ring. Throughout $\S 1, A$ will denote a commutative ring with identity.

1.1. The definition of the Riemann sphere of $A$. Two elements $s$ and $t$ of $A$ are said to have no common zero iff there is no proper ideal of $A$ which contains both $s$ and $t$. Clearly, "proper" may be replaced by "maximal" in the preceding definition without altering the meaning. It is also clear that $s$ and $t$ have no common zero iff there are $a, b$ in $A$ so that $s a+b t=1$.

Note that if $A=C, s$ and $t$ have no common zero iff $s$ and $t$ are not both 0 . If $A$ is $C[0,1], s$ and $t$ have no common zero iff there is no $x$ in $[0,1]$ at which $s(x)$ $=t(x)=0$.

For brevity, we shall often write " $(s, t)$ is admissible" in place of " $s$ and $t$ have no common zero." Let $S$ be the set of admissible ordered pairs $(s, t)$ of elements of $A$. We introduce a relation $R$ in $S$ by $(s, t) R(u, v)$ iff $s v=t u$. A basic description of $R$ is provided by

THEOREM 1.1.1. Let $(s, t)$ and $(u, v)$ be admissible. Then $(s, t) R(u, v)$ iff there is an invertible $x$ in $A$ so that $x s=u$ and $x t=v$.

Proof. One implication is trivial. Now assume that $(s, t) R(u, v)$. Choose $a, b$ in $A$ so that $a s+b t=1$. Then $u=u a s+b u t=(a u+b v) s$. Similarly $v=(a u+b v) t$. If $a u+b v$ is singular, then there is some proper ideal $I$ which contains $a u+b v$. But then the above equations show that $I$ contains both $u$ and $v$, which contradicts the admissibility of $(u, v)$. Therefore $x=a u+b v$ is invertible; furthermore $u=x s$ and $v=x t$.

That $R$ is an equivalence relation follows immediately from 1.1.1. We now define $A_{\infty}=$ the Riemann sphere of $A$, to be the set of all $R$-equivalence classes of $S$. If $(s, t)$ is admissible, the equivalence class to which it belongs will be denoted by $(s, t)$.

1.2. Algebraic operations on the Riemann sphere. The complex point at infinity is algebraically related to the finite complex numbers and to itself by the formulas $z+\infty=\infty, z \cdot \infty=\infty$ for $z \neq 0$, and $\infty \cdot \infty=\infty$. However, $\infty+\infty$ and $0 \cdot \infty$ are undefined. We will show that these notions generalize to the ring setting.

Let $D_{+}$be the set of ordered pairs $(p, q)$ of elements of $A_{\infty}$ so that if we write $p=(s, t)$ and $q=(u, v)$, then $(t, v)$ is admissible. Similarly, let $D^{\cdot}$ be the set of all $(p, q)$ in $A_{\infty} \times A_{\infty}$ so that if $p=(s, t)$ and $q=(u, v)$, then $(s, v)$ and $(u, t)$ are admissible. That these definitions, as well as those we shall make further on, do not depend on the choice of equivalence class representative, is an easy consequence of 1.1.1.

$D_{+}$and $D^{*}$ are actually the sets of those pairs of elements of $A_{\infty}$ which can be added and multiplied, respectively. More precisely, we have

TheOREM 1.2.1. Suppose $(s, t)$ and $(u, v)$ are admissible. Then $((s, t),(u, v))$ is in $D_{+}$iff $(s v+u t, t v)$ is admissible. Similarly, $((s, t),(u, v))$ is in $D^{\cdot}$ iff $(s u, t v)$ is admissible. 
The proof of 1.2.1 involves only elementary algebra, and is thus left to the reader.

1.2.1 enables us to define addition and multiplication on $D_{+}$and $D^{\cdot}$ respectively as follows: $+: D_{+} \rightarrow A_{\infty}$ is given by $(s, t)+(u, v)=(s v+u t, t v)$, while $\cdot: D^{\cdot} \rightarrow A_{\infty}$ is defined by $(s, t) \cdot(u, v)=(s u, t v)$. We summarize the basic algebraic properties of these operations with

THEOREM 1.2.2. Let $p, q$ and $r$ be elements in $A_{\infty}$. Then

(i) if $p+q(p \cdot q)$ is defined, then so is $q+p(q \cdot p)$, and $p+q=q+p(p \cdot q=q \cdot p)$,

(ii) if $(p+q)+r((p \cdot q) \cdot r)$ is defined, then so is $p+(q+r)(p \cdot(q \cdot r))$, and $(p+q)+r$ $=p+(q+r)((p \cdot q) \cdot r=p \cdot(q \cdot r))$,

(iii) $p+(0,1)(p \cdot(1,1))$ is defined and $=p$,

(iv) if $p \cdot q+p \cdot r$ is defined then so is $p \cdot(q+r)$, and $p \cdot q+p \cdot r=p(q+r)$.

Proof. We will prove (iv). Write $p=(s, t), q=(u, v)$ and $r=(x, y) \cdot p \cdot q+p \cdot r$ $=\left(s x t v+s u t y, t^{2} v y\right)$, so $t$ must be invertible. (If $t$ lies in some proper ideal $I$, then so do sxtv + suty and $t^{2} v y$, which is impossible, since they have no common zero.) A similar argument shows that $v$ and $y$ have no common zero. Hence by the definition of $D_{+}, q+r$ is defined and $=(u y+v x, v y)$. That $p \cdot(q+r)$ is defined and $=p \cdot q+p \cdot r$ now follows directly from 1.2.1.

In contrast with addition and multiplication, inverses are everywhere defined on $A_{\infty}$ by $(s, t)^{-1}=(t, s)$. The following theorem about inverses can be easily proved.

THEOREM 1.2.3. If $p$ is in $A_{\infty}$, then $\left(p^{-1}\right)^{-1}=p$. If $p$ and $q$ are in $A_{\infty}$, and $p \cdot q$ is defined, then $p^{-1} \cdot q^{-1}$ is defined and $=(p \cdot q)^{-1}$.

1.3. Th. fractional linear transformations. Unless otherwise specified, all $2 \times 2$ matrices will be assumed to have coefficients in $A$. The matrix $\left(\begin{array}{l}e f \\ g\end{array}\right)$ will be called regular iff the determinant $e h-g f$ is invertible in $A$. The regular $2 \times 2$ matrices form a group $G L(A)$ under matrix multiplication.

For each $T=\left(\begin{array}{ll}e & f \\ g & h\end{array}\right)$ in $G L(A)$, define $T^{\prime}: A_{\infty} \rightarrow A_{\infty}$ by $T^{\prime}(s, t)=(e s+f t, g s+h t)$. To show that $T^{\prime}$ is well defined, we must show that $e s+f t$ and gs $+h t$ have no common zero. Suppose $I$ is an ideal of $A$ which contains both $e s+f t$ and $g s+h t$. Then $g e s+g f t$ and egs $+e h t$ both lie in $I$; subtracting, we see that $(e h-g f) t$ is in $I$. Since $e h-g f$ is invertible, $t$ is in $I$. A similar argument shows that $I$ contains $s$. Since $(s, t)$ is admissible, $I=A$.

The fractional linear transformations of $A$ are defined to be those mappings of $A_{\infty}$ into itself which are of the form $T^{\prime}$, where $T$ is in $G L(A)$. The set of all fractional linear transformations of $A$ will be denoted by $G(A)$. Some fundamental properties of $G(A)$ are given by

THEOREM 1.3.1. Each fractional linear transformation $T^{\prime}$ is a bijection of $A_{\infty}$. $G(A)$ is the subgroup of the group (under composition), of all bijections of $A_{\infty}$. The mapping $T \rightarrow T^{\prime}$ is a group homomorphism of $G L(A)$ onto $G(A)$ whose kernel consists of those matrices of the form $\left(\begin{array}{ll}x & 0 \\ 0 & x\end{array}\right)$, where $x$ is an invertible element of $A$. 
Proof. We prove the assertion about the kernel. It is clear that if $x$ is invertible, $\left(\begin{array}{ll}x & 0 \\ 0 & x\end{array}\right)^{\prime}$ is the identity mapping of $A_{\infty}$. Now suppose that $T^{\prime}=\left(\begin{array}{ll}e & f \\ g & h\end{array}\right)^{\prime}$ is the identity mapping. Since $T^{\prime}(0,1)=(f, h)=(0,1), f=0$ and $h$ is invertible. Since $T^{\prime}(1,0)$ $=(e, g)=(1,0), g=0$ and $e$ is invertible. Since $T^{\prime}(1,1)=(e, h)=(1,1), e=h$.

We conclude this section with the essential

THEOREM 1.3.2. $G(A)$ acts transitively on $A_{\infty}$.

Proof. It is sufficient to show that for any $(s, t)$ in $A_{\infty}$, there is some f.l.t. $T^{\prime}$ which sends $(0,1)$ into $(s, t)$. Choose $a, b$ in $A$ so that $a s+b t=1$. Clearly $\left(\begin{array}{cc}-b & s \\ a & t\end{array}\right)^{\prime}(0,1)$ $=(s, t)$.

1.4. Mappings induced by ring homomorphisms. Let $A_{1}$ and $A_{2}$ be commutative rings with identity, $f: A_{1} \rightarrow A_{2}$ a ring (not necessarily a ring with identity) homomorphism. Write $j=f(1)$; clearly $j^{2}=j$.

Lemma 1.4.1. Suppose that $(s, t)$ is $A_{1}$-admissible and that $y$ is in $A_{2}$. Then $(f(s)+(1-j) y, f(t)+1-j)$ is $A_{2}$-admissible.

Proof. Choose $a, b$ in $A$ so that $a s+b t=1$. Then

$$
f(a)(f(s)+(1-j) y)+(j f(b)+1-j)(f(t)+1-j)=1 .
$$

1.4.1 shows that the function $f_{*}: A_{1 \infty} \rightarrow A_{2 \infty}$ given by $f_{*}(s, t)=(f(s), f(t)+1-j)$ is well defined. It also follows from 1.4.1 that if $(p, q)$ lies in $D_{1+}\left(D_{i}\right)$, then $\left(f_{*}(p), f_{*}(q)\right)$ is in $D_{2+}\left(D_{2}^{*}\right)$. A routine computation now proves

THEOREM 1.4.2. Let $p$ and $q$ be in $A_{1 \infty}$. If $p+q(p \cdot q)$ is defined, then $f_{*}(p)+f_{*}(q)$ $\left(f_{*}(p) \cdot f_{*}(q)\right)$ is defined and $=f_{*}(p+q)\left(f_{*}(p \cdot q)\right)$.

In view of $1.4 .2, f_{*}$ will be called the Riemann sphere homomorphism induced by $f$. It is important to note that the assignment of Riemann spheres $A_{\infty}$ to rings $A$, and Riemann sphere maps $f_{*}$ to ring maps $f$ is a covariant functor. In other words, a direct computation shows

THEOREM 1.4.3. If $f_{1}: A_{1} \rightarrow A_{2}$ and $f_{2}: A_{2} \rightarrow A_{3}$ are ring homomorphisms, then $\left(f_{2} \circ f_{1}\right)_{*}=f_{2^{*}} \circ f_{1 *}$. Furthermore, $\left(1_{A}\right)_{*}=1_{A_{\infty}}$.

$f$ also induces a group homomorphism from $G\left(A_{1}\right)$ into $G\left(A_{2}\right)$. To define this mapping, we need two lemmas.

Lemma 1.4.4. If $x$ is regular in $A_{1}$, then $y=f(x)+1-j$ is regular in $A_{2}$. If $T$ $=\left(\begin{array}{ll}a & b \\ c & d\end{array}\right)$ is in $G L\left(A_{1}\right)$, then

is in $G L\left(A_{2}\right)$.

$$
T_{f}=\left(\begin{array}{cc}
f(a)+1-j & f(b) \\
f(c) & f(d)+1-j
\end{array}\right)
$$

Proof. A simple computation shows that $y^{-1}$ is given by $f\left(x^{-1}\right)+1-j$. The second statement of 1.4 .3 now follows directly from the first. 
LEMMA 1.4.5. Suppose that $T$ and $U$ are in $G L\left(A_{1}\right)$, and that $T^{\prime}=U^{\prime}$. Then $T_{f}^{\prime}=U_{f}^{\prime}$.

Proof. By 1.3.1, there is an invertible $x$ in $A$ so that $x T=U$. Therefore

$$
(f(x)+1-j) T_{f}=U_{f} .
$$

\subsection{5 now follows from 1.3.1 and 1.4.3.}

Now we can define $f^{*}: G\left(A_{1}\right) \rightarrow G\left(A_{2}\right)$ as follows; if $T$ is in $G L\left(A_{1}\right)$, then $f^{*}\left(T^{\prime}\right)$ $=T_{f}^{\prime}$ 1.4.3 and 1.4.4 show that $f^{*}$ is well defined.

It is easy to verify that $f^{*}$ is a group homomorphism; $f^{*}$ will be called the group homomorphism induced by $f$. Again we have a covariant functor, this time assigning groups to rings, and group maps to ring maps. Explicitly, by a direct computation we have

THEOREM 1.4.6. If $f_{1}: A_{1} \rightarrow A_{2}$ and $f_{2}: A_{2} \rightarrow A_{3}$ are ring homomorphisms, then $\left(f_{2} \circ f_{1}\right)^{*}=f_{2}^{*} \circ f_{1}^{*}$. Furthermore $\left(1_{A}\right)^{*}=1_{G(A)}$.

The following relationship between $f_{*}$ and $f^{*}$, which can be directly verified, will be of vital importance.

THEOREM 1.4.7. Suppose $X$ is a fractional linear transformation of $A_{1}$. Then

$$
f^{*}(X) \circ f_{*}=f_{*} \circ X \text { and } f^{*}(X)^{-1} \circ f_{*} \circ X=f_{*} .
$$

1.5. The identification of $A$ with a subset of $A_{\infty}$. We now identify $A$ with a subset of $A_{\infty}$ via $a \leftrightarrow(a, 1)$. If $a$ and $b$ are in $A$, then $a+b$ and $a b$ are independent of whether the $A$-operations or the $A_{\infty}$-operations are being considered, as is $a^{-1}$ for invertible $a$. (From now on, for simplicity of notation, we will write $p q$ instead of $p \cdot q$ to denote the product of two elements of $A_{\infty}$.) However, if $a$ is a singular element of $A, a^{-1}$ is not defined in $A$, but is defined in $A_{\infty}$; there $a^{-1}=(1, a)$. From now on, for $a$ in $A, a^{-1}$ will denote the $A_{\infty}$-inverse of $a$. We can thus write

$$
A_{\infty}=\left\{a b^{-1} ;(a, b) \text { admissible, } a, b \text { in } A\right\} .
$$

Therefore the extension of $A$ to $A_{\infty}$ allows us in some sense to divide $a$ by $b$, whenever $a$ and $b$ are elements of $A$ with no common zero.

Now let $A_{1}, A_{2}$ and $f$ be as in 1.4. Via the above identification, $f_{*}$ is actually an extension of $f$ to $A_{1 \infty}$. If $(a, b)$ is $A_{1}$-admissible, then $f_{*}\left(a b^{-1}\right)=f(a)(f(b)+1-j)^{-1}$.

2. The Riemann sphere of a commutative Banach algebra. Throughout the remainder of this paper, $A$ will denote a complex commutative Banach algebra with identity. $A_{\infty}$ will denote the Riemann sphere of (the underlying ring of) $A$.

2.1. The definition and elementary properties of the topology for $A_{\infty}$. We now introduce a topology for $A_{\infty}$, by prescribing a neighborhood system $\mathscr{N}_{p}$ for each point $p$ of $A_{\infty}$. We define $\mathscr{N}_{p}$ to be the set of those subsets $N$ of $A_{\infty}$ which satisfy

(i) If $X$ is a f.l.t. which maps 0 into $p$, (such $X$ always exist because $G(A)$ is 
transitive), then there is an open neighborhood $U$ of 0 in $A$ so that $X(U)$ is contained in $N$.

Lemma 2.1.1. Let $X$ be a fractional linear transformation. Suppose that a and $X(a)$ both lie in $A$, and that $V$ is an open neighborhood of $X(a)$ in $A$. Then there is an open neighborhood $U$ of $a$ in $A$ so that $X(U) \subseteq V$.

Proof. Write $X=\left(\begin{array}{l}e \\ g\end{array}\right)^{\prime}$. Since $X(a)$ is in $A, g a+h$ is invertible. Choose a neighborhood $W$ of $a$ in $A$ so that $g b+h$ is invertible when $b$ lies in $W$. Clearly $X \mid W$ is a continuous mapping of $A$ into itself, so $U$ can be chosen to be an appropriate subset of $W$.

Lemma 2.1.2. Suppose that $X$ is a fractional linear transformation, and that $V$ is an open subset of $A$. If $p$ lies in $X(V)$, then $X(V)$ belongs to $\mathscr{N}_{p}$.

Proof. Let $Y$ be a f.l.t. which maps 0 into $p$. By 2.1.1, choose an open neighborhood $U$ of 0 in $A$ so that $X^{-1} \circ Y$ maps $U$ into $V$. Clearly $Y(U)$ is contained in $X(V)$, so $X(V) \in \mathscr{N}_{p}$.

We can now prove

THEOREM 2.1.3. The assignment $p \rightarrow \mathscr{N}_{p}$ defines a topology for $A_{\infty}$ in which for each $p, \mathscr{N}_{p}$ is the set of all neighborhoods of $p$.

Proof. All but the last of the standard neighborhood axioms are trivial to verify. Now let $p \in A_{\infty}$, and $N \in \mathscr{N}_{p}$. Choose a f.l.t. $X$ which sends 0 into $p$, and an open neighborhood $V$ of 0 in $A$ so that $X(V)$ is contained in $N$. By 2.1.2, if $q$ lies in $X(V), X(V) \in \mathscr{N}_{q}$.

From now on, $A_{\infty}$ will be considered to be provided with this topology. That this topology is the natural one for $A_{\infty}$ is made apparent by the following simple theorems.

THEOREM 2.1.4. If $U \subseteq A, U$ is $A$-open iff $U$ is $A_{\infty}$-open. Thus $A$ (with the usual norm topology) is an open subspace of $A_{\infty}$.

Proof. Let $U$ be $A$-open, set $X=$ the identity f.1.t. By 2.1.2, $X(U)=U$ is $A_{\infty}$-open.

Now let $U$ be $A_{\infty}$-open, let $u \in U$. Set $X=\left(\begin{array}{ll}1 & u \\ 0 & 1\end{array}\right)^{\prime}$, clearly $X(0)=u$. Therefore, there is some $\delta>0$ so that $X(B(0 ; \delta)) \subseteq U$; since $X(B(0 ; \delta))=B(u ; \delta), U$ is $A$-open.

THEOREM 2.1.5. Each fractional linear transformation is a homeomorphism of $A_{\infty}$.

Proof. It is sufficient to show that each f.l.t. $X$ is continuous at each $p \in A_{\infty}$. Let $N$ be a neighborhood of $X(p)$. Choose a f.l.t. $Y$ which sends 0 into $p$, and an open neighborhood $U$ of 0 in $A$ so that $X(Y(U)) \subseteq N$. Since $Y(U)$ is a neighborhood of $p$ in $A_{\infty}$ by 2.1.2, $X$ is continuous at $p$.

Note that the topology given $A_{\infty}$ is the unique topology for $A_{\infty}$ so that $A$ is an open subspace and each f.l.t. is a homeomorphism.

THEOREM 2.1.6. $A_{\infty}$ is a Hausdorff space. 
Proof. By 2.1.4 and 2.1.5, it is sufficient to separate 0 from $a b^{-1}$, where $a, b \in A$ and $b$ is singular. Set $U_{1}=B(0 ; 1)$ and $U_{2}=A_{\infty} \sim \bar{B}(0 ; 1) ; \bar{B}(0 ; 1)$ is $A_{\infty}$-closed by 2.1.4. Therefore $U_{1}$ and $U_{2}$ separate 0 and $a b^{-1}$.

2.2. Characterizations via the fractional linear group of the complex field and of semisimplicity. It is well known that the complex fractional linear transformations are 3-point transitive on the classical Riemann sphere. This phenomenon characterizes the complex field as a commutative complex Banach algebra with identity. In fact we have the stronger

THEOREM 2.2.1. $G(A)$ is 2-point transitive on $A_{\infty}$ iff $A$ is the complex field.

Proof. Suppose that 2-point transitivity holds in $A_{\infty}$. Let $a$ be a nonzero element of $A$. Choose a f.l.t. $X$ so that $X(a)=0$ and $X(0)=1$; write $X=\left(\begin{array}{c}e \\ g\end{array}\right)^{\prime}$. Since $X(0)=1$, $h$ is invertible, and $h=f$. Since $X(a)=0, g a+f$ is invertible and $e a+f=0$, thus $g a-e a$ is invertible, so $a$ is invertible. Therefore $A$ is a field, but the only complex Banach field is the complex field.

Notice that if $a, b \in A$, and $a$ is invertible, then $\left(\begin{array}{ll}a & b \\ 0 & 1\end{array}\right)^{\prime}$ maps $A$ into itself. Furthermore, if $r$ is in the radical of $A$, then $\left.\left(\begin{array}{l}1 \\ r\end{array}\right) \begin{array}{l}0 \\ 1\end{array}\right)^{\prime}$ also maps $A$ into itself. Fractional linear transformations of the latter kind are called radical transformations, and will play an important role later on. Fractional linear transformations of the former kind can be used to characterize semisimplicity via

THEOREM 2.2.2. A is semisimple iff every f.l.t. $X$ which maps $A$ into itself is of the form $\left(\begin{array}{l}a \\ 0 \\ 0\end{array}\right)$, where $a$ is invertible.

Proof. Assume that $A$ is semisimple, and that $X$ is a f.l.t. which sends $A$ into itself; write $X=\left(\begin{array}{c}e \\ g\end{array}\right)^{\prime}$. Since $X(0) \in A, h$ is invertible. Since for each nonzero complex $z, X(z)$ lies in $A, g z+h$ is invertible, so $h^{-1} g$ is in the radical. Since $A$ is semisimple, $h^{-1} g=0=g$. Therefore $X$ is of the form $\left(\begin{array}{ll}a & b \\ 0 & 1\end{array}\right)$, where $a=h^{-1} e$ and $b=h^{-1} f$.

Now assume that $A$ is not semisimple; let $r$ be a nonzero element of the radical. $\left(\begin{array}{ll}1 & 0 \\ r & 1\end{array}\right)^{\prime}$ maps $A$ into itself, and is not of the form $\left(\begin{array}{ll}a & b \\ 0 & 1\end{array}\right)^{\prime}$, , by 1.3.1.

2.3. The elementary topological quasi-algebra of the Riemann sphere. We begin with an important lemma, which uses the compactness of the maximal ideal space of $A$.

LEMMA 2.3.1. Suppose the elements of $A a_{0}$ and $b_{0}$ have no common zero. Then there is some $\delta>0$ so that if both $\left\|a-a_{0}\right\|$ and $\left\|b-b_{0}\right\|$ are $<\delta$, then $a$ and $b$ have no common zero.

Proof. Since $a_{0}$ and $b_{0}$ have no common zero, there is a positive number $3 \delta$ so that $\left|F\left(a_{0}\right)\right|+\left|F\left(b_{0}\right)\right| \geqq 3 \delta$, for all maximal ideals $F$. If both $\left\|a-a_{0}\right\|$ and $\left\|b-b_{0}\right\|$ are $<\delta$, then $|F(a)|+|F(b)| \geqq \delta$ for all maximal ideals $F$, so $a$ and $b$ have no common zero.

We will now show that the topology for $A_{\infty}$ blends nicely with the algebra of $A_{\infty}$ introduced in $\$ 1$. 
TheOREM 2.3.2. $D_{+}$is open. + is a continuous mapping of $D_{+}$into $A_{\infty}$.

Proof. Let $s, t, u$ and $v$ be elements of $A$ so that $(s, t)$ and $(u, v)$ are admissible, and $\left(s t^{-1}, u v^{-1}\right)$ lies in $D_{+}$. Then $(t, v)$ is admissible. Choose $e, f, g$ and $h$ from $A$ so that $-e s+f t=1=-g u+h v$; write $X_{1}=\left(\begin{array}{ll}f & s \\ e & t\end{array}\right)^{\prime}$ and $X_{2}=\left(\begin{array}{ll}h & u \\ g & v\end{array}\right)^{\prime} ;$ clearly $X_{1}(0)=s t^{-1}$ and $X_{2}(0)=u v^{-1}$. By 2.3.1 choose $\delta_{1}>0$ so that $(e a+t, g b+v)$ is admissible when both $a$ and $b$ lie in $B\left(0 ; \delta_{1}\right)$. Therefore

so $D_{+}$is open.

$$
X_{1}\left(B\left(0 ; \delta_{1}\right)\right) \times X_{2}\left(B\left(0 ; \delta_{1}\right)\right) \subseteq D_{+},
$$

Now choose $X_{3}=\left(\begin{array}{cc}q & r \\ x & y\end{array}\right)^{\prime}$ so that $X_{3}\left(s t^{-1}+u v^{-1}\right)=0$. Define $\phi: B\left(0 ; \delta_{1}\right) \times B\left(0 ; \delta_{1}\right)$ $\rightarrow A_{\infty}$ via $\phi(a, b)=X_{3}\left(X_{1}(a)+X_{2}(b)\right)$. To show that + is continuous on $D_{+}$, it is sufficient to show that $\phi$ is continuous at $(0,0)$.

For $i=1, \ldots, 4$ define $\psi_{i}: B\left(0 ; \delta_{1}\right) \times B\left(0 ; \delta_{1}\right) \rightarrow A$ via

$$
\begin{aligned}
& \psi_{1}(a, b)=(g b+v)(f a+s)+(e a+t)(h b+u), \\
& \psi_{2}(a, b)=(e a+t)(g b+v), \\
& \psi_{3}(a, b)=q \psi_{1}(a, b)+r \psi_{2}(a, b), \text { and } \\
& \psi_{4}(a, b)=x \psi_{1}(a, b)+y \psi_{2}(a, b) .
\end{aligned}
$$

Clearly all the $\psi_{i}$ are continuous. A direct computation shows that $\phi(a, b)=$ $\psi_{3}(a, b) \psi_{4}(a, b)^{-1}$ for $(a, b)$ in $B\left(0 ; \delta_{1}\right) \times B\left(0 ; \delta_{1}\right)$. Since $\phi(0,0)=0, \psi_{4}(0,0)$ is invertible; choose $\delta_{2}$ so that $0<\delta_{2}<\delta_{1}$ and $\psi_{4}(a, b)$ is invertible when $(a, b)$ lies in $B\left(0 ; \delta_{2}\right) \times B\left(0 ; \delta_{2}\right)$. Thus on $B\left(0 ; \delta_{2}\right) \times B\left(0 ; \delta_{2}\right), \phi$ is the quotient (in $\left.A\right)$ of two continuous functions; so $\phi$ is continuous on $B\left(0 ; \delta_{2}\right) \times B\left(0 ; \delta_{2}\right)$.

COROLlary 2.3.3. Let $Y$ be a topological space, and let $f_{1}, \ldots, f_{n}$ be continuous maps of $Y$ into $A_{\infty}$ so that if $y \in Y$ and $i \neq j$, then $\left(f_{i}(y), f_{j}(y)\right)$ is in $D_{+}$. Then $f_{1}+\cdots$ $+f_{n}$ is defined and continuous on $Y$.

Proof. That $f_{1}+\cdots+f_{n}$ is defined (and is independent of the way parentheses are associated in its definition) follows from 1.2.2. (ii) and the remark that $(a, b c)$ is admissible when $(a, b)$ and $(a, c)$ are admissible. The continuity of $f_{1}+\cdots+f_{n}$ follows from 2.3.2.

By arguments analogous with those used for + , we can prove

THEOREM 2.3.4. $D^{*}$ is open, and $\cdot$ is a continuous mapping of $D^{*}$ into $A_{\infty}$. If $Y$ is a topological space, and $f_{1}, \ldots, f_{n}$ are continuous mappings of $Y$ into $A_{\infty}$ so that when $i \neq j, f_{i}(y) f_{j}(y)$ is defined for each $y$, then $f_{1} \cdots f_{n}$ is a well-defined continuous map of $Y$ into $A_{\infty}$.

Note that it is trivial to show that $p \rightarrow p^{-1}$ is continuous on $A_{\infty}$, since ${ }^{-1}=\left(\begin{array}{ll}0 & 1 \\ 1 & 0\end{array}\right)^{\prime}$. An interesting application of this, and the preceding ideas, is provided by

TheOREM 2.3.5. The invertible elements of $A$ are dense in $A$ iff $A$ is dense in $A_{\infty}$.

Proof. Assume the invertible elements of $A$ are not dense in $A$. Choose an open set $U$ in $A$ so that $U$ contains only singular elements. Then $\left(\begin{array}{ll}0 & 1 \\ 1 & 0\end{array}\right)^{\prime}(U)$ is open in $A_{\infty}$ and does not intersect $A$. 
Now assume $A$ is not dense in $A_{\infty}$. Choose an open set $V$ in $A_{\infty}$ which does not meet $A$. Let $p$ be an element of $V$, write $p=s t^{-1}$, where $s$ and $t$ are elements of $A$ with no common zero. By 2.3.1 there is some $\delta_{1}>0$ so that $(s, b)$ is admissible when $\|b-t\|<\delta_{1}$. Define $\phi: B\left(t ; \delta_{1}\right) \rightarrow A_{\infty}$ via $\phi(b)=s b^{-1}$. Since $\phi$ is continuous, there is some $\delta_{2}>0$ so that $\phi(b)$ is not in $A$ when $\|b-t\|<\delta_{2}$. Thus $B\left(t ; \delta_{2}\right)$ does not intersect the invertible elements of $A$.

THEOREM 2.3.6. Let $f: A_{1} \rightarrow A_{2}$ be a ring homomorphism of the underlying rings of $A_{1}$ and $A_{2}$; if $f$ is continuous then so is $f_{*}$.

Proof. Apply the continuity of $f_{*}$ at 0 , and 1.4.6.

COROLlARY 2.3.7. If $A_{1}$ and $A_{2}$ are topologically and algebraically isomorphic, then $A_{1 \infty}$ and $A_{2 \infty}$ are homeomorphic.

Proof. Let $f: A_{1} \rightarrow A_{2}$ and $g: A_{2} \rightarrow A_{1}$ be continuous ring homomorphisms so that $g \circ f=1\left(A_{1}\right)$ and $f \circ g=1\left(A_{2}\right)$. By the covariance of ${ }_{*}, g_{*} \circ f_{*}=1\left(A_{1 \infty}\right)$, $f_{*} \circ g_{*}=1\left(A_{2 \infty}\right)$, and $f_{*}$ and $g_{*}$ are continuous by 2.3.6.

2.4. Definition and elementary properties of the Gelfand transform and the spectrum of elements of $A_{\infty}$. Let $F$ be a maximal ideal of $A$, i.e. a continuous algebra homomorphism of $A$ onto $C$. By 2.3.6 F lifts to a continuous "algebraic" homomorphism $F_{*}$ of $A_{\infty}$ onto $C_{\infty}=$ the extended complex plane. Now let $p$ be an element of $A_{\infty}$. Define $\hat{p}$, the Gelfand transform of $p$, to be the mapping of $\mathscr{M}$ into $C_{\infty}$ defined by $\hat{p}(F)=F_{*}(p)$. This definition clearly agrees with the usual definition when $p \in A$.

To see that $\hat{p}$ is continuous on $\mathscr{M}$, write $p=a b^{-1}$, where $a$ and $b$ are elements of $A$ with no common zero. A direct computation shows that $\hat{p}=\hat{a}(\hat{b})^{-1}$, which is continuous by 2.3.4.

Now define the spectrum of $p$ (written $\sigma(p)$ ) to be $\hat{p}(\mathscr{M})$. Since $\mathscr{M}$ is compact and $p$ is continuous, $\sigma(p)$ is a compact set in the extended plane. If $p$ lies in $A$, this notion of spectrum agrees with the usual definition.

Observe that $\infty$ belongs to the spectrum of $p$ iff $p$ is not in $A$. A proof runs as follows; obviously if $p \in A, \sigma(p)$ is contained in the finite plane. If $p$ is not in $A$, write $p=a b^{-1}$ as above; $b$ is singular. Let $F$ be a maximal ideal so that $F(b)=0$, clearly $F_{*}(p)=\infty$.

With this definition of spectrum, and 1.4 .7 , a routine computation proves the following spectral mapping theorem for complex fractional linear transformations.

THEOREM 2.4.1. Suppose $X$ is a fractional linear transformation on $A$ which is of the form $\left(\begin{array}{c}e \\ g\end{array} h\right)^{\prime}$, where $e, f, g$ and $h$ are complex numbers. Then $\sigma(X(p))=X(\sigma(p))$.

We will need later not only the continuity of $F \rightarrow \hat{p}(F)$ and $p \rightarrow \hat{p}(F)$, but the stronger

THEOREM 2.4.2. The mapping $\phi: \mathscr{M} \times A_{\infty} \rightarrow C_{\infty}$ defined by $\phi(F, p)=\hat{p}(F)$ is continuous. 
Proof. We will show continuity at $\left(F_{0}, p_{0}\right)$. Choose $X=\left(\begin{array}{l}e \\ g \\ h\end{array}\right)^{\prime}$ from $G(A)$ so that $X(0)=p_{0}$. Define $\psi: \mathscr{M} \times A_{\infty} \rightarrow \mathscr{M} \times A_{\infty}$ via $\psi(F, p)=(F, X(p))$; since $\psi$ is a homeomorphism and $\psi\left(F_{0}, 0\right)=\left(F_{0}, p_{0}\right)$ it is sufficient to show that $\phi \circ \psi$ is continuous at $\left(F_{0}, 0\right)$. Since $F_{0}^{*}(X)^{-1}$ is a homeomorphism of $C_{\infty}$ it is sufficient to show that $\theta=F_{0}^{*}(X)^{-1} \circ \phi \circ \psi$ is continuous at $\left(F_{0}, 0\right)$.

Define $\xi_{1}$ and $\xi_{2}$ to be the maps of $\mathscr{M} \times A$ into $C$ given by

$$
\begin{aligned}
& \xi_{1}(F, a)=F_{0}(h) F(e a+f)+F_{0}(-f) F(g a+h), \\
& \xi_{2}(F, a)=F_{0}(-g) F(e a+f)+F_{0}(e) F(g a+h) .
\end{aligned}
$$

Since $\phi \mid \mathscr{M} \times A$ is continuous, $\xi_{1}$ and $\xi_{2}$ are continuous. A direct computation shows that when $(F, a)$ lies in $\mathscr{M} \times A$,

$$
\theta(F, a)=\xi_{1}(F, a) \xi_{2}(F, a)^{-1}
$$

Thus by 2.3.4, $\theta \mid \mathscr{M} \times A$ is continuous, so $\phi$ is continuous at $\left(F_{0}, 0\right)$.

2.5. Finite products of Riemann spheres and compactness. Recall that if $A_{1}, \ldots, A_{n}$ are commutative Banach algebras with identity, their direct sum is defined to be

$$
A_{1} \oplus \cdots \oplus A_{n}=\left\{\left(a_{1}, \ldots, a_{n}\right): a_{i} \in A_{i}\right\},
$$

where the algebraic operations are pointwise, and

$$
\left\|\left(a_{1}, \ldots, a_{n}\right)\right\|=\sup \left\|a_{i}\right\| \text {. }
$$

$A_{1} \oplus \cdots \oplus A_{n}$ is also a commutative Banach algebra with identity. We will show that the identity mapping of $A_{1} \oplus \cdots \oplus A_{n}$ can be lifted to a natural homeomorphism of the Riemann sphere of $A_{1} \oplus \cdots \oplus A_{n}$ with the product of the Riemann spheres of the $A_{i}$.

For $1 \leqq i \leqq n$ define $P_{j}: A_{1} \oplus \cdots \oplus A_{n} \rightarrow A_{j}$ and $Q_{j}: A_{j} \rightarrow A_{1} \oplus \cdots \oplus A_{n}$ by

$$
P_{j}\left(a_{1}, \ldots, a_{n}\right)=a_{j}, \quad Q_{j}\left(a_{j}\right)=\left(0, \ldots, a_{j}, \ldots, 0\right) .
$$

$P_{j}$ and $Q_{j}$ are both continuous ring homomorphisms so that

$$
P_{j} \circ Q_{j}=1\left(A_{j}\right) \text {, and } P_{k} \circ Q_{j}=0 \text { when } j \neq k \text {. }
$$

We state without proof the simple and well-known

LEMMA 2.5.1. If $F_{j}$ is a maximal ideal of $A_{j}$, then $F_{j} \circ P_{j}$ is a maximal ideal of $A_{1} \oplus \cdots \oplus A_{n}$. Conversely, if $F$ is a maximal ideal of $A_{1} \oplus \cdots \oplus A_{n}$, then there is some $j$, and a maximal ideal $F_{j}$ of $A_{j}$ so that $F=F_{j} \circ P_{j}$.

THEOREM 2.5.2. The mappings $P_{\#}:\left(A_{1} \oplus \cdots \oplus A_{n}\right)_{\infty} \rightarrow A_{1 \infty} \times \cdots \times A_{n \infty}$ and $Q_{\#}: A_{1 \infty} \times \cdots \times A_{n \infty} \rightarrow\left(A_{1} \oplus \cdots \oplus A_{n}\right)_{\infty}$ defined below satisfy the conditions

$P_{\#}$ and $Q_{\#}$ are homeomorphisms so that $Q_{\#}=\left(P_{\#}\right)^{-1}$,

$P_{\#}\left|A_{1} \oplus \cdots \oplus A_{n}=Q_{\#}\right| A_{1} \oplus \cdots \oplus A_{n}=1\left(A_{1} \oplus \cdots \oplus A_{n}\right)$. 
Proof. $P_{j}$ and $Q_{j}$ lift to continuous "algebraic" maps $P_{j^{*}}:\left(A_{1} \oplus \cdots \oplus A_{n}\right)_{\infty}$ $\rightarrow A_{j \infty}$ and $Q_{j^{*}}: A_{j \infty} \rightarrow\left(A_{1} \oplus \cdots \oplus A_{n}\right)_{\infty}$ via 1.4.2 and 2.3.6. 1.4.3 shows that $P_{j^{*}} \circ Q_{j^{*}}=1\left(A_{j \infty}\right)$. Let $\pi_{j}$ be the continuous projection of $A_{1 \infty} \times \cdots \times A_{n \infty}$ onto $A_{j \infty}$. We need the following technical lemma.

LEMMA 2.5.3. If $i \neq j$ and $x \in A_{1 \infty} \times \cdots \times A_{n \infty}$, then $Q_{i^{*}}\left(\pi_{i}(x)\right)+Q_{j^{*}}\left(\pi_{j}(x)\right)$ is defined in $\left(A_{1} \oplus \cdots \oplus A_{n}\right)_{\infty}$.

Proof (of 2.5.3). If 2.5 .3 is not true, then there is some maximal ideal $F$ of $A_{1} \oplus \cdots \oplus A_{n}$ so that

$$
F_{*}\left(Q_{i}\left(\pi_{i}(x)\right)\right)=F_{*}\left(Q_{j^{*}}\left(\pi_{j}(x)\right)\right)=\infty .
$$

Choose $K$ so that $1 \leqq K \leqq n$, and there is a maximal ideal $F_{K}$ of $A_{K}$ so that $F=F_{K} \circ P_{K}$; we may assume without loss of generality that $K \neq i$. But now

$$
\infty=F_{*} \circ Q_{i^{*}} \circ \pi_{i}(x)=F_{K^{*}} \circ P_{K^{*}} \circ Q_{i^{*}} \circ \pi_{i}(x)=F_{K^{*}} \circ\left(P_{k} \circ Q_{i}\right)_{*} \circ \pi_{i}(x)=0,
$$

which is impossible, so 2.5 .3 is proved.

We now return to the proof of 2.5.2. By 2.5.3 and 2.3.4, we can define $Q_{\#}: A_{1 \infty} \times \cdots \times A_{n \infty} \rightarrow\left(A_{1} \oplus \cdots \oplus A_{n}\right)_{\infty}$ via

$$
Q_{\#}(x)=\sum_{i=1}^{n} Q_{i \bullet}\left(\pi_{i}(x)\right) \text {. }
$$

$Q_{\#}$ is continuous on its domain. Now define $P_{\#}$ to be the mapping of $\left(A_{1} \oplus \cdots \oplus A_{n}\right)_{\infty}$ into $A_{1 \infty} \times \cdots \times A_{n \infty}$ given by

$$
P_{\#}(p)=\left(P_{1^{*}}(p), \ldots, P_{n^{*}}(p)\right) \text {. }
$$

$P_{\#}$ is also continuous. But

$$
\begin{aligned}
P_{\#} \circ Q_{\#}\left(p_{1}, \ldots, p_{n}\right) & =\left(P_{m^{*}}\left(\sum_{i=1}^{n} Q_{i \cdot}\left(p_{i}\right)\right)\right)_{m=1}^{n} \\
& =\left(\sum_{i=1}^{n}\left(P_{m} \circ Q_{i}\right)_{*}\left(p_{i}\right)\right)_{m=1}^{n}=\left(p_{1}, \ldots, p_{n}\right) .
\end{aligned}
$$

Furthermore

$$
\begin{aligned}
Q_{\#} \circ P_{\#}\left(a b^{-1}\right) & =Q_{\#} \circ P_{\#}\left(\left(a_{1}, \ldots, a_{n}\right)\left(b_{1}, \ldots, b_{n}\right)^{-1}\right) \\
& =Q_{\#}\left(a_{1} b_{1}^{-1}, \ldots, a_{n} b_{n}^{-1}\right)=\sum_{i=1}^{n} Q_{i \bullet}\left(a_{i} b_{i}^{-1}\right) \\
& =\left(\sum_{i=1}^{n} Q_{i}\left(a_{i}\right)\right)\left(\sum_{i=1}^{n} Q_{i}\left(b_{i}\right)+1-\sum_{i=1}^{n} Q_{i}(1)\right)^{-1}=a b^{-1} .
\end{aligned}
$$

Thus $P_{\#} \circ Q_{\#}$ and $Q_{\#} \circ P_{\#}$ are both identity mappings, so $P_{\#}$ and $Q_{\#}$ are homeomorphisms and $Q_{\#}=\left(P_{\#}\right)^{-1}$. It is clear that $P_{\#}$ and $Q_{\#}$ leave $A_{1} \oplus \cdots \oplus A_{n}$ fixed. 
Evidence of the naturality of $P_{\#}$ and $Q_{\#}$ is provided by

THEOREM 2.5.4. The functions $P^{\#:}: G\left(A_{1} \oplus \cdots \oplus A_{n}\right) \rightarrow G\left(A_{1}\right) \times \cdots \times G\left(A_{n}\right)$ and $Q^{\#:} G\left(A_{1}\right) \times \cdots \times G\left(A_{n}\right) \rightarrow G\left(A_{1} \oplus \cdots \oplus A_{n}\right)$ defined below satisfy the conditions $P^{\#}$ and $Q^{\#}$ are group isomorphisms so that $Q^{\#}=\left(P^{\#}\right)^{-1}$.

If $X \in G\left(A_{1} \oplus \cdots \oplus A_{n}\right)$, then $P^{\#}(X) \circ P_{\#}=P_{\#} \circ X$.

Proof. Via 1.4, we have the group homomorphisms

$$
P_{j}^{*}: G\left(A_{1} \oplus \cdots \oplus A_{n}\right) \rightarrow G\left(A_{j}\right) \quad \text { and } \quad Q_{j}^{*}: G\left(A_{j}\right) \rightarrow G\left(A_{1} \oplus \cdots \oplus A_{n}\right) .
$$

Define $P^{\#}: G\left(A_{1} \oplus \cdots \oplus A_{n}\right) \rightarrow G\left(A_{1}\right) \times \cdots \times G\left(A_{n}\right)$ by

$$
P^{\#}(X)=\left(P_{1}^{*}(X), \ldots, P_{n}^{*}(X)\right) \text {. }
$$

Define $Q^{\#}: G\left(A_{1}\right) \times \cdots \times G\left(A_{n}\right) \rightarrow G\left(A_{1} \oplus \cdots \oplus A_{n}\right)$ by

$$
Q^{\#}\left(X_{1}, \ldots, X_{n}\right)=Q_{1}^{*}\left(X_{1}\right) \cdots Q_{n}^{*}\left(X_{n}\right) \text {. }
$$

Obviously $P^{\#}$ is a group homomorphism. Furthermore, a direct computation shows that $Q^{\#} \circ P^{\#}$ and $P^{\#} \circ Q^{\#}$ are identity maps, thus $Q^{\#}$ is a group isomorphism and $Q^{\#}=\left(P^{\#}\right)^{-1}$.

Now let $X$ be in $G\left(A_{1} \oplus \cdots \oplus A_{n}\right)$. By 1.4.7 $P_{j}^{*}(X) \circ P_{j^{*}}=P_{j^{*}} \circ X$. It follows easily that $P^{\#}(X) \circ P_{\#}=P_{\#} \circ X$.

The preceding discussion of products enables us to answer the question: when is $A_{\infty}$ compact? Note that $A_{\infty}$ is locally compact iff $A$ is locally compact iff the underlying vector space of $A$ is finite dimensional.

THEOREM 2.5.5. $A_{\infty}$ is compact iff $A$ is algebraically isomorphic to the product of $n$ complex planes.

Proof. Let $f: A \rightarrow C^{n}$ and $g: C^{n} \rightarrow A$ be complex algebra homomorphisms so that $g \circ f=1(A)$ and $f \circ g=1\left(C^{n}\right)$. Since $A$ is finite dimensional, $f$ and $g$ are both continuous. Thus $f_{*}$ and $g_{*}$ provide a homeomorphism of $A_{\infty}$ and $C_{\infty}^{n}$; but $C_{\infty}^{n}$ is compact by 2.5.2. (In fact, $C_{\infty}^{n}$ is homeomorphic to the product of $n$ 2-spheres.) Therefore $A_{\infty}$ is compact.

Let $A_{\infty}$ be compact, by the remark preceding the statement of 2.5 .5 , the underlying vector space of $A$ has finite dimension $N$. Suppose $A$ has $N+1$ distinct maximal ideals $F_{1}, \ldots, F_{N+1}$. Set $\mathscr{M}^{\prime}=\left\{F_{1}, \ldots, F_{N+1}\right\}$, define $\phi: A \rightarrow C\left(\mathscr{M}^{\prime}\right)$ by $\phi(a)=\hat{a} \mid \mathscr{M}^{\prime} . \phi$ is a homeomorphism of $A$ onto $C\left(\mathscr{M}^{\prime}\right)$, which contradicts $\operatorname{dim} C\left(\mathscr{M}^{\prime}\right)$ $\leqq \operatorname{dim} A$. Thus $A$ has exactly $n$ distinct maximal ideals, where $n \leqq N$.

Suppose $r$ is in the radical of $A$, and $r \neq 0$. Since $A_{\infty}$ is compact, the sequence $m r$, $m=1,2, \ldots$ has a cluster point $p$ in $A_{\infty}$. Let $F$ be a maximal ideal, since $F_{*}$ is continuous, $F_{*}(p)=0$. Therefore $\sigma(p)=\{0\}$, so $p$ lies in $A$, which is clearly impossible. We conclude that $A$ is semisimple, and $\wedge$ is a topological isomorphism of $A$ onto $C(\mathscr{M}) \approx C^{n}$. 
2.6. The connected components of the Riemann sphere. Elements of $A_{\infty}$ whose spectrum is the whole extended plane. The principal connected component of $A_{\infty}$ is the connected component of $A_{\infty}$ which contains $A$. It will be denoted by $A_{\infty p}$.

Note that all the components of $A_{\infty}$ are homeomorphic, since $G(A)$ is transitive, and each f.l.t. is a homeomorphism. Since $A_{\infty}$ is locally connected, each component of $A_{\infty}$ is open in $A_{\infty}$.

THEOREM 2.6.1. Suppose $p$ is an element of $A_{\infty}$ whose spectrum is a proper subset of the extended plane. Then $p$ belongs to the principal component of $A_{\infty}$.

Proof. Choose $z \neq 0$ from $C_{\infty}$ so that $z$ is not in $\sigma(p)$. Let $X$ be a complex fractional linear transformation which sends 0 into 0 and $z$ into $\infty$. By 2.4.1 $\infty \notin \sigma(X(p))$, so $X(p)$ lies in $A$, and therefore in $A_{\infty p}$. Since $X(0)$ and $X(p)$ lie in $A_{\infty p}, 0$ and $p$ belong to the same component of $A_{\infty}$; thus $p \in A_{\infty p}$.

We now give two examples of elements $p$ of $A_{\infty}$ whose spectrum is $C_{\infty}$. In the first example, $p$ lies in $A_{\infty p}$, while in the second $p$ does not. Thus there are algebras whose Riemann sphere is disconnected.

EXAmple 2.6.2. Let $A=C$ (the finite complex plane $R^{2}$ ). Define $f, g: R^{2} \rightarrow C$ via $f(z)=z$ when $|z| \leqq 1, f(z)=z /|z|$ when $|z| \geqq 1, g(z)=1$ when $|z| \leqq 1, g(z)=1 /|z|$ when $|z| \geqq 1$. Clearly $f$ and $g$ are in $C\left(R^{2}\right)$. Let $I$ be an ideal of $C\left(R^{2}\right)$ which contains both $f$ and $g$, then $h=f \bar{f}+g$ is in $I$; since $|h(z)| \geqq 1$ for all $z, h$ is invertible, so $I=A$. Therefore $f$ and $g$ have no common zero, set $p=f g^{-1} \in A_{\infty}$. For $w \in R^{2}$, let $F_{w}$ be the maximal ideal of $A$ given by evaluation at $w$; clearly $F_{w^{*}}(p)=f(w) g(w)^{-1}=w$. Thus the spectrum of $p$ contains the finite plane; since the spectrum is compact $\sigma(p)=C_{\infty}$. Now set $X=\left(\frac{g}{f}-f\right)^{\prime} \in G(A) . X(p)=0$ and $X(0)=-f$, so $X(p)$ and $X(0)$ are in $A_{\infty p}$. Thus 0 and $p$ lie in the same component of $A_{\infty} ; p \in A_{\infty p}$.

The preceding example can be used to answer negatively a question raised by Blum [1, p. 359]. Blum asks: If $p(a)=x_{0}+x_{1} a+\cdots+x_{n} a^{n}$ is a polynomial with coefficients in a commutative complex Banach algebra with identity $A$, and $p(a)$ is singular for all $a$ in $A$, is there a maximal ideal $F$ of $A$ so that $F\left(x_{j}\right)=0$, all $j$ ? Let $A, f, g$ and $h$ be as in 2.6.2; define $p(a)=g a-f$. We have already shown that there is no proper ideal $I$ which contains both $f$ and $g$; we will show that $p(a)$ is singular for each $a$ in $A$. Let $a \in A$, choose $r \geqq 0$ so that the range of $a$ is contained in the closed complex disc of radius $r$. By the Brouwer fixed point theorem, the restriction of $a$ to $\bar{K}(0 ; r)$ has a fixed point $z^{*}$. Thus $p(a)\left(z^{*}\right)=0$, so $p(a)$ is singular.

We precede the second example with two lemmas.

LEMMA 2.6.3. Let $\phi$ be a continuous $A_{\infty}$-valued function defined on $[0,1]$. Let a and $b$ be elements of $A$ with no common zero so that $\phi(0)=a b^{-1}$. Then there are two continuous $A$-valued functions $\psi_{1}$ and $\psi_{2}$ defined on $[0,1]$ so that

(i) If $0 \leqq t \leqq 1, \psi_{1}(t) \psi_{2}(t)^{-1}$ is defined in $A_{\infty}$ and $=\phi(t)$, and

(ii) $\psi_{1}(0)=a, \psi_{2}(0)=b$.

Proof. That $\psi_{1}$ and $\psi_{2}$ can be defined in a neighborhood of 0 so as to satisfy 
(i) and (ii) follows from writing $\phi(t)=X^{-1}(X(\phi(t)))$, where $X$ is a f.l.t. which sends $\phi(0)$ into 0 , and then applying 1.1.1. A standard extension argument now completes the proof.

LeMma 2.6.4. Let $\psi_{1}$ and $\psi_{2}$ be continuous A-valued functions defined on $[0,1]$ so that

(i) For each $t, \psi_{1}(t) \psi_{2}(t)^{-1}$ is defined in $A_{\infty}$, and

(ii) $\psi_{1}(1) \psi_{2}(1)^{-1}=0$.

Then $\psi_{1}$ and $\psi_{2}$ can be extended to continuous A-valued functions (also called $\psi_{1}$ and $\psi_{2}$ ) on $[0,3]$ so that (i) is satisfied, and $\psi_{1}(3)=\psi_{2}(3)=1$.

Proof. If $1 \leqq t \leqq 2$ set $\psi_{1}(t)=t-1$ and $\psi_{2}(t)=\psi_{2}(1)$. If $2 \leqq t \leqq 3$ set $\psi_{1}(t)=1$ and $\psi_{2}(t)=(t-2)+(3-t) \psi_{2}(1)$.

EXAMPLE 2.6.5. Set $A=C\left(S^{3}\right)$, where

$$
S^{3}=\text { the 3-sphere }=\left\{\left(z_{1}, z_{2}\right):\left|z_{1}\right|^{2}+\left|z_{2}\right|^{2}=1, z_{1}, z_{2} \text { complex }\right\} .
$$

Define $f$ and $g: S^{3} \rightarrow C$ via $f(z, w)=z$ and $g(z, w)=w ; f$ and $g$ are in $A$. $f$ and $g$ clearly have no common zero; write $p=f g^{-1} \in A_{\infty}$.

Suppose that $p$ lies in the principal component of $A_{\infty}$. Then there is a continuous map $\phi$ of $[0,1]$ into $A_{\infty p}$ so that $\phi(0)=p$ and $\phi(1)=0$. By 2.6.3 and 2.6 .4 choose continuous $A$-valued functions $\psi_{1}^{*}$ and $\psi_{2}^{*}$ defined on $[0,1]$ so that

(i) If $0 \leqq t \leqq 1,\left(\psi_{1}^{*}(t), \psi_{2}^{*}(t)\right)$ is admissible,

(ii) $\psi_{1}^{*}(0)=f_{0}, \psi_{2}^{*}(0)=g_{0}$, and

(iii) $\psi_{1}^{*}(1)=1=\psi_{2}^{*}(1)$.

Define $\psi_{1}, \psi_{2}: S^{3} \times[0,1] \rightarrow C$ by $\psi_{i}(x, t)=\psi_{i}^{*}(t)(x) ; \psi_{1}$ and $\psi_{2}$ are continuous. Define $\psi_{3}: S^{3} \times[0,1] \rightarrow S^{3}$ via

$$
\psi_{3}=\left(\psi_{1} /\left(\left.\left|\psi_{1} !^{2}+\right| \psi_{2}\right|^{2}\right)^{1 / 2}, \psi_{2} /\left(\left|\psi_{1}\right|^{2}+\left|\psi_{2}\right|^{2}\right)^{1 / 2}\right) .
$$

$\psi_{3}(, 0): S^{3} \rightarrow S^{3}$ is the identity map, while $\psi_{3}(, 1): S^{3} \rightarrow S^{3}$ maps $S^{3}$ onto the point $(1 / \sqrt{ } 2,1 / \sqrt{ } 2)$. Thus the identity map of $S^{3}$ is homotopic to the constant map on $S^{3}$, contradicting the well-known noncontractability of $S^{3}$. Therefore $p$ does not lie in the principal component of $A_{\infty}$. 2.6.1 shows that the spectrum of $p$ is the extended plane. Furthermore, the Riemann sphere of $A$ is not connected.

\section{Abstract analytic function theory.}

3.1. The quotient of a holomorphic function by a maximal ideal. Suppose that $D$ is a domain in $A, f: D \rightarrow A$ is holomorphic, i.e. analytic in the sense of Lorch [6, p. 417], on $D$, and $F$ is a maximal ideal of $A$. If there is a (necessarily unique) complex holomorphic function $g$ defined on the complex domain $F(D)$ so that $g \circ F=F \circ f$ on $D$, we say $g$ is the quotient function of $f$ with respect to $F$, and write $g=f_{F}$.

This fundamental concept, introduced in [6] is investigated in detail in [4]. In particular, it is shown there that if $D$ is star shaped, $f_{F}$ exists. However, an example is given of a simply connected $D$, a holomorphic $f$ on $D$, and a maximal ideal $F$ so 
that $f_{F}$ does not exist. Here we will present only those basic results required in the sequel.

Notice that if $D$ is a ball $B(0 ; s), f_{F}$ is always defined. In fact, if $f$ is given by the power series $f(a)=\sum_{n} c_{n} a^{n}, f_{F}$ is defined on $K(0 ; s)$ by $f_{F}(z)=\sum_{n} F\left(c_{n}\right) z^{n}$.

It is simple, but important, to notice also that if $f$ and $g$ are holomorphic on $A$, then $(g \circ f)_{F}=g_{F} \circ f_{F}$. Furthermore $1(A)_{F}=1(C)$.

THEOREM 3.1.1. Let $f$ be holomorphic on $A$, and let $H$ be the complex entire functions with the compact open topology. Define $\phi: \mathscr{M} \rightarrow H$ by $\phi(F)=f_{F}$. Then $\phi$ is a continuous mapping.

Proof. Let $F_{0}$ be a maximal ideal. To show the continuity of $\phi$ at $F_{0}$, we must show that for any two positive numbers $R$ and $\varepsilon$, there is a neighborhood $U$ of $F_{0}$ in $\mathscr{M}$ so that

$$
\left|f_{F}(z)-f_{F_{0}}(z)\right|<\varepsilon,
$$

whenever $F$ lies in $U$ and $|z| \leqq R$. Choose $N>0$ so that

$$
\sum_{n=N+1}^{\infty}\left\|c_{n}\right\| R^{n}<\varepsilon / 3
$$

Set $U=\left\{F:\left|F\left(c_{n}\right)-F_{0}\left(c_{n}\right)\right|<\varepsilon / 3(n+1) R^{n}, n=0, \ldots, N\right\}$. If $F \in U$ and $|z| \leqq R$, then

$$
\begin{aligned}
\left|f_{F}(z)-f_{F_{0}}(z)\right| & \leqq \sum_{n>N+1}\left|F\left(c_{n}\right)\right| R^{n}+\sum_{n=0}^{N}\left|F\left(c_{n}\right)-F_{0}\left(c_{n}\right)\right| R^{n}+\sum_{n>N+1}\left|F_{0}\left(c_{n}\right)\right| R^{n} \\
& <\varepsilon / 3+\varepsilon / 3+\varepsilon / 3=\varepsilon .
\end{aligned}
$$

3.2. The definition and elementary properties of meromorphic functions. Just as in the case $A=C$, we can speak of an "analytic" function $f$ defined on an open set $D$ in $A_{\infty}$ and taking values in $A_{\infty}$ if we use $G(A)$ to define "local coordinates" on $A_{\infty}$. Such functions will be called meromorphic. A formal definition runs as follows: let $D$ be an open set in $A_{\infty}$, let $f$ be an $A_{\infty}$-valued function on $D$, and $p$ be a point of $D$. $f$ is meromorphic at $p$ iff there are $U, X_{1}$ and $X_{2}$ so that

(i) $U$ is an open connected neighborhood of $p$ in $D$,

(ii) $X_{1}$ and $X_{2}$ are fractional linear transformations so that $X_{1}(U)$ and $X_{2}(f(U)$ ) are both subsets of $A$,

(iii) $X_{2} \circ f \circ X_{1}^{-1}: X_{1}(U) \rightarrow A$ is holomorphic at $X_{1}(p)$. $f$ is meromorphic on $D$ iff it is meromorphic at each point of $D$.

In connection with the above definition, the following remarks are in order. Meromorphic functions are continuous. If $D$ is a domain in $A$, and $f$ is $A$-valued, then $f$ is meromorphic iff it is holomorphic. Each fractional linear transformation is meromorphic on $A_{\infty}$. The composition of meromorphic functions is again meromorphic.

THEOREM 3.2.1. Let $D$ be a domain in $A, f_{1}$ and $f_{2}$ holomorphic functions on $D$ so that $f_{1}(a) f_{2}(a)^{-1}$ is defined in $A_{\infty}$ for each $a$ in $D$ (or equivalently, $f_{1}(a)$ and $f_{2}(a)$ have no common zero for each $a$ in $D$ ). Then $h(a)=f_{1}(a) f_{2}(a)^{-1}$ is meromorphic on $D$. 
Proof. Let $a_{0} \in D$. Choose a f.l.t. $X=\left(\begin{array}{ll}e & b \\ c & d\end{array}\right)^{\prime}$ so that $X\left(h\left(a_{0}\right)\right)=0$. Since $h$ is continuous by 2.3 .4 , choose $\delta>0$ so that $U=B\left(a_{0} ; \delta\right) \subseteq D$ and $X(h(U)) \subseteq A$. If $a \in U$,

$$
X(h(a))=\left(e f_{1}(a)+b f_{2}(a)\right)\left(c f_{1}(a)+d f_{2}(a)\right)^{-1} \in A .
$$

Thus the restriction of $X \circ h$ to $U$ is the quotient in $A$ of holomorphic functions and is thus itself holomorphic. It now follows from the remarks preceding the theorem that $h$ is meromorphic.

We do not know if the global converse of 3.2.1 is valid. However, we can prove the local converse, i.e.

THEOREM 3.2.2. Let $D$ be a domain in $A, h$ meromorphic on $D, a_{0}$ a point of $D$. Then there is some open neighborhood $U$ of $a_{0}$ in $D$, and holomorphic functions $f_{1}$ and $f_{2}$ defined on $U$ so that $f_{1}(a) f_{2}(a)^{-1}$ is defined and equal to $h(a)$ for each $a$ in $U$.

Proof. Choose a f.l.t. $X$ which sends $h\left(a_{0}\right)$ into 0 , write $X^{-1}=\left(\begin{array}{ll}e & b \\ c & d\end{array}\right)^{\prime}$. Let $U$ be an open neighborhood of $a_{0}$ in $D$ so that $X(h(U)) \subseteq A$; for $i=0,1,2$ define $f_{i}$ on $U$ by $f_{0}(a)=X(h(a)), f_{1}(a)=e f_{0}(a)+b$, and $f_{2}(a)=c f_{0}(a)+d$. All the $f_{i}$ are clearly holomorphic; if $a \in U, f_{1}(a) f_{2}(a)^{-1}$ is defined and $=h(a)$.

The preceding results enable us to prove

THEOREM 3.2.3. Let $D$ be a domain in $A, h_{1}$ and $h_{2}$ meromorphic functions on $D$ so that $h(a)=h_{1}(a)+h_{2}(a)$ is defined in $A_{\infty}$ for each $a$ in $D$. Then $h$ is meromorphic on $D$.

Proof. Let $a_{0} \in D$. By 3.2.2 choose an open neighborhood $U$ of $a_{0}$ in $D$, and holomorphic functions $f_{1}, f_{2}, g_{1}$ and $g_{2}$ on $U$ so that $h_{1}(a)=f_{1}(a) f_{2}(a)^{-1}$ and $h_{2}(a)$ $=g_{1}(a) g_{2}(a)^{-1}$ for each $a$ in $U$. Then the restriction of $h$ to $U$ is given by

$$
\left(g_{2} f_{1}+f_{2} g_{1}\right)\left(f_{2} g_{2}\right)^{-1}
$$

hence $h$ is meromorphic at $a_{0}$ by 3.2.1.

It is immediate from 3.2.4 that the sum, when defined, of finitely many meromorphic functions on a domain $D$ in $A$ is again meromorphic. That the product, when defined, of finitely many meromorphic functions is again meromorphic follows from an argument analogous to that given for sums. The restriction that $D$ lie in $A$, rather than in $A_{\infty}$, can be easily removed. Finally, observe that since the operation of taking inverses is given by a fractional linear transformation, the inverse of a meromorphic function is always defined and meromorphic.

Theorem 3.2.4 (IDentity Theorem). Let $D$ be a domain in $A_{\infty}, h_{1}$ and $h_{2}$ meromorphic on D. If $h_{1}$ and $h_{2}$ agree on an open subset of $D$, then $h_{1}$ and $h_{2}$ agree on $D$.

Proof. By a standard connectedness argument, the observation that locally a meromorphic function can be written $X_{2} \circ f \circ X_{1}$, where $X_{1}$ and $X_{2}$ are f.1.t.'s and $f$ is holomorphic, and the identity theorem for holomorphic functions. 
3.3. Simple analytic polynomials and simple algebraic polynomials. A basic theorem of classical function theory is that a complex entire function $f(z)$ is a polynomial iff $\lim _{z \rightarrow \infty} f(z)$ exists in $C_{\infty}$. Our task here is that of generalizing this theorem to the Lorch analytic function theory. With this in mind we formulate the following definitions:

An analytic polynomial (an.p.) on $A$ is a holomorphic function $f: A \rightarrow A$ so that $\lim _{z \rightarrow \infty, z \in C} f(z)$ (or briefly, $\lim _{z \rightarrow \infty} f(z)$ ) exists in $A_{\infty}$.

A simple analytic polynomial (s.an.p.) on $A$ is an analytic polynomial $f$ so that if $f(a)=\sum_{n} c_{n} a^{n}$ is the power series expansion of $f$ on $A$, there is some $K>0$ so that $c_{K}$ is invertible, and $c_{n}$ lies in the radical for $n>K . K$ is called the degree of $f$.

In this subsection, we will describe the simple analytic polynomials on $A$. In 3.5 we will describe the analytic polynomials on $A$.

Let $S$ denote the class of all $A_{\infty}$-valued functions defined on $A_{\infty}$. Let $S_{Y}$ denote the class of all those functions $f$ in $S$ so that $p f(p)$ is defined for all $p$ in $A_{\infty}$. Define $Y: S_{Y} \rightarrow S$ by $Y(f)(p)=p f(p)$.

For each $a$ in $A$, define $T_{a}$ (translation by $a$ ) to be the fractional linear transformation $\left(\begin{array}{ll}1 & a \\ 0 & 1\end{array}\right)^{\prime}$. Define $\bar{T}_{a}: S \rightarrow S$ by $\bar{T}_{a}(f)=T_{a} \circ f$.

For each $r$ in the radical of $A$, define $R_{r}$ (the radical transformation induced by $r$ ) by $R_{r}=\left(\begin{array}{cc}1 & 0 \\ r & 1\end{array}\right)^{\prime}$. Define $\bar{R}_{r}: S \rightarrow S$ by $\bar{R}_{r}(f)=R_{r} \circ f$.

For each $a$ in $A$ and $r$ in the radical of $A$, define $Z(a, r): S_{Y} \rightarrow S$ by $Z(a, r)(f)$ $=\bar{R}_{r} \circ \bar{T}_{a} \circ Y(f)$.

LeMma 3.3.1. If $f$ lies in $S_{Y}$, so does $Z(a, r)(f)$.

Proof. Let $p \in A_{\infty}$, write $p=a_{1} b_{1}^{-1}, f(p)=a_{2} b_{2}^{-1}$, where for $i=1,2\left(a_{i}, b_{i}\right)$ is an admissible pair. Then

$$
\left(\bar{R}_{r} \circ \bar{T}_{a} \circ Y(f)\right)(p)=\left(a_{1} a_{2}+a b_{1} b_{2}\right)\left(b_{1} b_{2}+r a_{1} a_{2}+r a b_{1} b_{2}\right)^{-1} .
$$

We must verify that

$$
q=\left(a_{1} b_{1}^{-1},\left(a_{1} a_{2}+a b_{1} b_{2}\right)\left(b_{1} b_{2}+r a_{1} a_{2}+r a b_{1} b_{2}\right)^{-1}\right) \in D .
$$

Suppose that $F$ is a maximal ideal, and

$$
F\left(a_{1} a_{2}+a b_{1} b_{2}\right)=F\left(b_{1}\right)=0 .
$$

Then $F\left(a_{1} a_{2}\right)=0$, since $a_{1}$ and $b_{1}$ have no common zero $F\left(a_{2}\right)$ is 0 , contradicting $(p, f(p)) \in D^{-}$. Thus $a_{1} a_{2}+a b_{1} b_{2}$ and $b_{1}$ have no common zero. Now suppose that $F$ is a maximal ideal so that

$$
F\left(b_{1} b_{2}+r a_{1} a_{2}+r a b_{1} b_{2}\right)=F\left(a_{1}\right)=0 .
$$

Since $r$ is in the radical, $F\left(b_{1} b_{2}\right)=0$; since $\left(a_{1}, b_{1}\right)$ is admissible $F\left(b_{2}\right)=0$, contradicting $(p, f(p)) \in D^{*}$. Therefore $a_{1}$ and $b_{1} b_{2}+r a_{1} a_{2}+r a b_{1} b_{2}$ have no common zero, hence $q$ lies in $D^{\circ}$. 
THEOREM 3.3.2. Let $x_{0}$ be an invertible element of $A$, let $a_{1}, \ldots, a_{K}$ be elements of $A$, and let $r_{1}, \ldots, r_{K}$ be elements of the radical of $A$. Define $f_{0}$ on $A_{\infty}$ by $f_{0}\left(A_{\infty}\right)=x_{0}$; clearly $f_{0}$ lies in $S_{Y}$. For $1 \leqq j \leqq K$ write $Z_{j}=Z\left(a_{j}, r_{j}\right)$. Set

$$
f=Z_{K} \circ Z_{K-1} \circ \cdots \circ Z_{1}\left(f_{0}\right) \text {. }
$$

Then $f$ is defined and meromorphic on $A_{\infty}$, and $f$ maps $A$ into itself. Furthermore, the restriction of $f$ to $A$ is a simple analytic polynomial of degree $K$.

Proof. 3.3.1 shows that $f$ is defined on $A_{\infty}$; that $f$ is meromorphic on $A_{\infty}$ follows from the elementary properties of meromorphic functions. It is clear that $f$ maps $A$ into itself.

We will show that $f$ is a s.an.p. of degree $K$ by induction on $K$. The case $K=0$ is trivial. For $K>0$, set $f_{1}=Z_{K-1} \circ \cdots \circ Z_{1}\left(f_{0}\right)$, and let $\sum d_{n} a^{n}$ be the power series expansion of $f_{1} \mid A$. By the inductive hypothesis $d_{K-1}$ is invertible, and $d_{n}$ is in the radical for $n>K-1$. Define $f_{2}: A \rightarrow A$ by $f_{2}(a)=a f_{1}(a)$. Obviously $f_{2}$ is a s.an.p. of degree $K$. It is also easy to see that $f_{3}$ is a s.an.p. of degree $K$, where $f_{3}$ is defined on $A$ by $f_{3}(a)=f_{2}(a)+a_{K}$. Finally, define $f_{4}: A \rightarrow A$ by

$$
f_{4}(a)=f_{3}(a)\left(r_{K} f_{3}(a)+1\right)^{-1}
$$

If $F$ is a maximal ideal, $F\left(f_{4}(a)\right)=F\left(f_{3}(a)\right)$, thus $f_{4 F}=f_{3 F}$. Therefore $f \mid A=f_{4}$ is a s.an.p. of degree $K$.

Any meromorphic function $f$ on $A_{\infty}$ which can be constructed from an invertible $x_{0}$, arbitrary $a_{1}, \ldots, a_{K}$, and $r_{1}, \ldots, r_{K}$ in the radical in the way described in 3.3.2, will be called a simple algebraic polynomial (s.al.p.) of degree $K$. Under this terminology 3.3.2 says that the restriction of a simple algebraic polynomial of degree $K$ to $A$ is a simple analytic polynomial of degree $K$.

Note that any polynomial (in the usual sense) on $A$ with invertible leading coefficient can be extended to an s.al.p. Conversely, if $A$ is semisimple and $f$ is an s.al.p., then the restriction of $f$ to $A$ is a polynomial on $A$ with invertible leading coefficient. Thus the construction of a simple algebraic polynomial on $A$ is a generalization of the construction of a complex polynomial, as well as a way of producing simple analytic polynomials.

TheOREM 3.3.3 (UNIQUENESS OF THE CONSTRUCTION OF A S.AL.P.). Let $f$ be the s.al.p. constructed from $x_{0}, a_{1}, \ldots, a_{K}$ and $r_{1}, \ldots, r_{K}$, and let $g$ be the s.al.p. constructed from $x_{0}^{\prime}, a_{1}^{\prime}, \ldots, a_{J}^{\prime}$ and $r_{1}^{\prime}, \ldots, r_{J}^{\prime}$. If $f|A=g| A$, then $K=J, x_{0}=x_{0}^{\prime}, a_{j}=a_{j}^{\prime}$ and $r_{j}=r_{j}^{\prime}$ for all $j$.

Proof. Since $f \mid A$ and $g \mid A$ are s.an.p.s of degree $K$ and $J$ respectively, $K=J$. We now proceed by induction on $K$. The case $K=0$ is trivial. Now suppose $K>0$, write $Z_{j}=Z\left(a_{j}, r_{j}\right)$ and $Z_{j}^{\prime}=Z\left(a_{j}^{\prime}, r_{j}^{\prime}\right)$. Set

$$
f_{1}=Z_{K-1} \circ \cdots \circ Z_{1}\left(f_{0}\right) \text { and } g_{1}=Z_{K-1}^{\prime} \circ \cdots \circ Z_{1}^{\prime}\left(g_{0}\right) \text {. }
$$


Let $\infty$ denote $(1,0) \in A_{\infty}$. Then (writing $a_{j}=a(j)$ and $r_{j}=r(j)$ ) $f(\infty)=$ $R_{r(K)} \circ T_{a(K)}\left(\infty f_{1}(\infty)\right)=R_{r(K)} \circ T_{a(K)}(\infty)=R_{r(K)}(\infty)=r_{K}^{-1}$. Similarly $g(\infty)=\left(r_{K}^{\prime}\right)^{-1}$, since $f(\infty)=g(\infty), r_{K}=r_{K}^{\prime}$. Furthermore,

$$
R_{-r(K)}(f(0))=T_{a(K)}\left(0 f_{1}(0)\right)=T_{a(K)}(0)=a_{K} .
$$

Applying the same argument to $g(0)=f(0)$, we find that $a_{K}=a_{K}^{\prime}$. Therefore $a f_{1}(a)$ $=a g_{1}(a)$ for all invertible $a$, so $f_{1}=g_{1}$ on $A$. An application of the inductive hypothesis completes the proof.

We can now prove the important

THEOREM 3.3.4. Let $g$ be a simple analytic polynomial. Then there is a unique simple algebraic polynomial $f$ whose restriction to $A$ is $g$.

Proof. Let $\sum c_{n} a^{n}$ be the power series expansion of $g$ on $A . c_{K}$ is invertible, where $K=$ degree $g$, and $c_{n}$ lies in the radical for $n>K$. Set $q=\lim _{z \rightarrow \infty} g(z)$. We proceed by induction on $K$. If $K=0$, and $F$ is a maximal ideal, $F_{*}(q)=\lim _{z \rightarrow \infty} F(g(z))=F\left(c_{0}\right)$. Thus $q$ lies in $A$; by Liouville's theorem $g$ is identically $q$.

Now consider $K>0$. If $F$ is a maximal ideal,

$$
F_{*}(q)=\lim _{z \rightarrow \infty} F\left(c_{0}\right)+F\left(c_{1}\right) z+\cdots+F\left(c_{K}\right) z^{K}=\infty .
$$

Thus the spectrum of $q$ contains only the point at infinity, so we can write $q=s^{-1}$, where $s$ is in the radical of $A$.

Set $b=g(0)(1-s g(0))^{-1}$, consider the function $g_{1}$ on $A$ defined by $g_{1}$ $=T_{-b} \circ R_{-s} \circ g$. Clearly $g_{1}$ is holomorphic on $A, g_{1}(0)=0$, and $\lim _{z \rightarrow \infty} g_{1}(z)=\infty$. It is not hard to see that if $\sum_{n=1}^{\infty} d_{n} a^{n}$ is the power series expansion of $g_{1}$ on $A$, and $F$ is a maximal ideal, then $F\left(c_{n}\right)=F\left(d_{n}\right), n \geqq 1$. Thus $g_{1}$ is an s.an.p. of degree $K$.

Now define $g_{2}$ on $A$ by $g_{2}(a)=\sum_{n=0}^{\infty} d_{n+1} a^{n}$. Since $\lim _{z \rightarrow \infty} g_{1}(z)=\infty$,

$$
\lim _{z \rightarrow 0}\left(g_{1}(1 / z)\right)^{-1}=0,
$$

so $\lim _{z \rightarrow 0}\left(g_{1}(1 / z)\right)^{-1} / z$ exists in $A$. Therefore $\lim _{z \rightarrow \infty} z g_{1}(z)^{-1}$ exists in $A$, so by the continuity of ${ }^{-1} \lim _{z \rightarrow \infty} g_{1}(z) / z=g_{2}(z)$ exists in $A_{\infty}$. Therefore $g_{2}$ is a s.an.p. of degree $K-1$. By the inductive hypothesis there is a s.al.p. $f_{2}$ so that $f_{2} \mid A=g_{2}$. Now clearly $f=Z(b, s)\left(f_{2}\right)$ is a s.al.p. whose restriction to $A$ is $g$. The uniqueness of $f$ follows from 3.3.3.

3.4. The meromorphic automorphisms of the principal component of the Riemann sphere. As an application of 3.3.4, we will show that each meromorphic automorphism of $A_{\infty p}$ is given by a fractional linear transformation.

Lemma 3.4.1. Let $\mathscr{Y}:[0,1] \rightarrow C_{\infty}$ be a curve, let $p_{0} \in A_{\infty}$. Suppose $F$ is a maximal ideal which sends $p_{0}$ into $\mathscr{Y}(0)$. Then there is a curve $\gamma:[0,1] \rightarrow A_{\infty}$ so that $\gamma(0)=p_{0}$ and $F_{*} \circ \gamma=\mathscr{Y}$.

Proof. If range $\mathscr{Y}$ lies in $C$, we can define $\gamma$ by

$$
\gamma(t)=p_{0}+\mathscr{Y}(t)-F_{*}\left(p_{0}\right)
$$


If range $\mathscr{Y}$ is a proper subset of $C_{\infty}$, choose a complex f.l.t. $X$ so that $\mathscr{Y}^{\prime}=X \circ \mathscr{Y}$ is a curve in $C$. Since $F_{*}\left(X\left(p_{0}\right)\right)=\mathscr{Y}^{\prime}(0)$, there is a curve $\gamma^{\prime}:[0,1] \rightarrow A_{\infty}$ so that $\gamma^{\prime}(0)=X\left(p_{0}\right)$ and $F_{*} \circ \gamma^{\prime}=\mathscr{Y}^{\prime}$. Set $\gamma=X^{-1} \circ \gamma^{\prime} ; \gamma(0)=p_{0}$, and

$$
F_{*} \circ \gamma=F_{*} \circ X^{-1} \circ \gamma^{\prime}=X^{-1} \circ F_{*} \circ \gamma^{\prime}=\mathscr{Y} \text {. }
$$

If range $\mathscr{Y}=C_{\infty}, \gamma$ can be constructed by applying the preceding construction on suitable subintervals of $[0,1]$.

LemMA 3.4.2. Let $g$ be meromorphic on $A_{\infty p}$, let $F$ be a maximal ideal. Then there is a (necessarily unique) complex meromorphic function $g_{F}$ on $C_{\infty}$ so that $g_{F} \circ F_{*}$ $=F_{*} \circ g \cdot g_{F}$ is called the quotient function of $g$ with respect to $f$.

Proof. Let $p$ be in $A_{\infty}$. Choose fractional linear transformations $X_{1}$ and $X_{2}$ so that $X_{1}(0)=p$ and $X_{2}(g(p))=0$. Now choose $t>0$ so that $f=\left(X_{2} \circ g \circ X_{1}\right) \mid B(0 ; t)$ is holomorphic. Let $f_{F}: K(0 ; t) \rightarrow C$ be the quotient function of $f$ by $F$.

Set $U=X_{1}(B(0 ; t)) ; U$ is an open connected neighborhood of $p$ in $A_{\infty}$. Note that $F_{*}(U)=F^{*}\left(X_{1}\right)(K(0 ; t))$ is an open connected neighborhood of $F_{*}(p)$ in $C_{\infty}$. Define $g_{F_{p}}: F_{*}(U) \rightarrow C_{\infty}$ by

$$
g_{F_{p}}=F^{*}\left(X_{2}^{-1}\right) \circ f_{F} \circ F^{*}\left(X_{1}^{-1}\right) .
$$

If $q \in U$, two applications of 1.4 .7 show that

$$
g_{F_{p}} \circ F_{*}(q)=F_{*} \circ g(q) \text {. }
$$

Set $\phi_{F_{p}}=$ the complex analytic element $\left\{g_{F_{p}}, F_{*}(p)\right\}$ (recall that our notions of complex analytic element, continuation, etc. are taken from Saks and Zygmund [10]). (1) shows that $\phi_{F_{p}}$ is determined uniquely by $F$ and $p$, i.e. is independent of the choice of $X_{1}, X_{2}$ and $t$. Set

$$
\mathscr{F}=\left\{\phi_{F_{p}}: p \in A_{\infty p}\right\} .
$$

Observe that if $\gamma:[0,1] \rightarrow A_{\infty p}$ is a curve in $A_{\infty p}$ it follows from (1) that $\left\{\phi_{F \gamma(t)}\right\}_{0 \leqq t \leqq 1}$ is a complex analytic chain along $F_{*} \circ \gamma$. Thus any two elements of $\mathscr{F}$ are continuations of each other.

Now let $q \in A_{\infty p}$, and let $\mathscr{Y}:[0,1] \rightarrow C_{\infty}$ be a curve starting at $F_{*}(q)$. By 3.4.1 let $\gamma:[0,1] \rightarrow A_{\infty}$ be a curve starting at $q$ so that $F_{*} \circ \gamma=\mathscr{Y} .\left\{\phi_{F \%(t)}\right\}_{0 \leqq t \leqq 1}$ is a continuation of $\phi_{F_{q}}$ along $\gamma$ consisting of elements of $\mathscr{F}$. Thus $\mathscr{F}$ is a complex analytic function, arbitrarily continuable in $C_{\infty}$.

By the monodromy theorem, $\mathscr{F}$ defines a complex meromorphic function $g_{F}: C_{\infty} \rightarrow C_{\infty}$. If $p \in A_{\infty p}$,

$$
g_{F} \circ F_{*}(p)=g_{F_{p}} \circ F_{*}(p)=F_{*} \circ g(p) .
$$

So $g_{F} \circ F_{*}=F_{*} \circ g$ on $A_{\infty p}$.

THEOREM 3.4.3. Let $g$ be a meromorphic automorphism of $A_{\infty p}$, i.e., $g$ is a meromorphic bijection of $A_{\infty p}$ whose inverse is meromorphic. Then there is a unique fractional linear transformation $X$ whose restriction to $A_{\infty p}$ is $g$. 
Proof. Since $G(A)$ is transitive, we may assume without loss of generality that $g(\infty)=\infty$. Let $h$ be the inverse function of $g$; for each maximal ideal $F$ form the quotient functions $g_{F}$ and $h_{F}$. If $z \in C$,

$$
g_{F} \circ h_{F}(z)=g_{F} \circ h_{F} \circ F_{*}(z)=F_{*} \circ g \circ h(z)=z .
$$

Similarly $h_{F} \circ g_{F}(z)=z$, so $g_{F}$ is a complex meromorphic automorphism of $C_{\infty}$ whose inverse is $h_{F}$.

But

$$
g_{F}(\infty)=g_{F} \circ F_{*}(\infty)=F_{*} \circ g(\infty)=\infty .
$$

Hence $g_{F}(z) \neq \infty$ if $z$ is a finite complex number. So if $a \in A, g_{F} \circ F_{*}(a)=F_{*} \circ g(a)$ is finite, which implies that $g$ maps $A$ into $A$. Let $\sum c_{n} a^{n}$ be the power series for $g$ on $A$, then $\sum F\left(c_{n}\right) z^{n}$ is the power series for $g_{F}$ on $C$. Since $g_{F}$ is a complex f.l.t. which leaves $\infty$ fixed, $F\left(c_{1}\right) \neq 0$ and $F\left(c_{n}\right)=0$ for $n>1$. Thus $g \mid A$ is a simple analytic polynomial of degree 1 .

By 3.3.4 there is a simple algebraic polynomial $f$ of degree 1 whose restriction to $A$ is $g$. But any s.al.p. of degree 1 is a f.l.t. $X$. Since $X=g$ on $A, X=g$ on $A_{\infty p}$.

The uniqueness of $X$ follows from the remark that if $X_{1}$ is a f.l.t. which leaves 0,1 and $\infty$ fixed, $X_{1}$ must be the identity transformation.

3.5. Analytic polynomials and algebraic polynomials. In 3.3 it was shown that any simple analytic polynomial is the restriction to $A$ of a simple algebraic polynomial. We will define an algebraic polynomial to be a finite direct sum (in an appropriate sense) of simple algebraic polynomials. Then we will show that each analytic polynomial can be thought of as a "finite direct sum" of simple analytic polynomials, and thus is the restriction to $A$ of an algebraic polynomial.

LEMMA 3.5.1. Let $A_{1}, \ldots, A_{n}$ be commutative complex Banach algebras with identity, $h_{1}, \ldots, h_{n}$ meromorphic functions on $A_{1 \infty}, \ldots, A_{n \infty}$. Define $h_{*}: A_{1 \infty} \times \ldots$ $\times A_{n \infty} \rightarrow A_{1 \infty} \times \cdots \times A_{n \infty}$ by

$$
h_{*}\left(p_{1}, \ldots, p_{n}\right)=\left(h_{1}\left(p_{1}\right), \ldots, h_{n}\left(p_{n}\right)\right) \text {. }
$$

Define $h^{*}:\left(A_{1} \oplus \cdots \oplus A_{n}\right)_{\infty} \rightarrow\left(A_{1} \oplus \cdots \oplus A_{n}\right)_{\infty}$ by (see 2.5.2 for the definitions of $P_{\#}$ and $\left.Q_{\#}\right)$

$$
h^{*}(p)=Q_{\#} \circ h_{*} \circ P_{\#}(p) .
$$

$h^{*}$ is meromorphic on $\left(A_{1} \oplus \cdots \oplus A_{n}\right)_{\infty}$.

Proof. Let $p \in\left(A_{1} \oplus \cdots \oplus A_{n}\right)_{\infty}$, we will show that $h^{*}$ is meromorphic at $p$. Set $P_{\#}(p)=\left(p_{1}, \ldots, p_{n}\right)$. For each integer $j$ so that $1 \leqq j \leqq n$ choose

(1) $X_{j 1} \in G\left(A_{j}\right)$ so that $X_{j 1}\left(p_{j}\right)=0$,

(2) $X_{j 2} \in G\left(A_{j}\right)$ so that $X_{j 2}\left(h_{j}\left(p_{j}\right)\right)=0$,

(3) $\delta_{j}>0$ so that $X_{j 2} \circ h_{j} \circ X_{j 1}^{-1}\left(B\left(0 ; \delta_{j}\right)\right) \subseteq A_{j}$, and set $\delta_{0}=\inf _{1 \leqq j \leqq n} \delta_{j}$. Thus $X_{j 2} \circ h_{j} \circ X_{j 1}^{-1}$ is holomorphic on $B\left(0 ; \delta_{j}\right)$; let 
$\sum_{k=0}^{\infty} c_{j k}\left(a_{j}\right)^{k}$ be its power series expansion there. Set (see 2.5.4 for the definition of $Q^{\#)}$

$$
X_{1}=Q^{*}\left(X_{11}, \ldots, X_{n 1}\right) \text { and } X_{2}=Q^{*}\left(X_{12}, \ldots, X_{n 2}\right)
$$

$X_{1}$ and $X_{2}$ lie in $G\left(A_{1} \oplus \cdots \oplus A_{n}\right)$. Set $U=X_{1}^{-1}\left(B\left(0 ; \delta_{0}\right)\right)$.

Clearly $U$ is open and connected, and $X_{1}$ maps $U$ into $A$. We will show that $p \in U$ by showing that $X_{1}(p)=0$.

$$
\begin{aligned}
X_{1}(p) & =Q^{\#}\left(X_{11}, \ldots, X_{n 1}\right) \circ Q_{\#} \circ P_{\#}(p) \\
& =\left(\text { by 2.5.4) } Q_{\#}\left(X_{11}, \ldots, X_{n 1}\right) \circ P_{\#}(p)=0 .\right.
\end{aligned}
$$

Now let $a=\left(a_{1}, \ldots, a_{n}\right) \in B\left(0 ; \delta_{0}\right)$. Then

$$
\begin{aligned}
& X_{2} \circ h^{*} \circ X_{1}^{-1}(a)=X_{2} \circ h^{*} \circ Q^{\#}\left(X_{11}^{-1}, \ldots, X_{n 1}^{-1}\right) \circ Q_{\#}\left(a_{1}, \ldots, a_{n}\right) \\
&=(\text { by } 2.5 .4) X_{2} \circ h^{*} \circ Q_{\#} \circ\left(X_{11}^{-1}, \ldots, X_{n 1}^{-1}\right)\left(a_{1}, \ldots, a_{n}\right) \\
&=X_{2} \circ Q_{\#} \circ h_{*} \circ P_{\#} \circ Q_{\#}\left(X_{11}^{-1}\left(a_{1}\right), \ldots, X_{n 1}^{-1}\left(a_{n}\right)\right) \\
&=Q^{\#}\left(X_{12}, \ldots, X_{n 2}\right) \circ Q_{\#}\left(h_{1} \circ X_{11}^{-1}\left(a_{1}\right), \ldots, h_{n} \circ X_{n 1}^{-1}\left(a_{n}\right)\right) \\
&=\left(\text { since } X_{j 2} \circ h_{j} \circ X_{j 1}^{-1} \text { lies in } A_{j} \text { for all } j\right) \\
&\left(X_{12} \circ h_{1} \circ X_{11}^{-1}\left(a_{1}\right), \ldots, X_{n 2} \circ h_{n} \circ X_{n 1}^{-1}\left(a_{n}\right)\right)=\sum_{k=0}^{\infty} c_{k} a^{k},
\end{aligned}
$$

where $c_{k}=\left(c_{1 k}, \ldots, c_{n k}\right)$. Thus $X_{2} \circ h^{*} \circ X_{1}^{-1}$ is represented on $B\left(0 ; \delta_{0}\right)$ by the convergent power series $\sum c_{k} a^{k}$, so $X_{2} \circ h^{*} \circ X_{1}^{-1}$ is holomorphic at 0 . Therefore $h^{*}$ is meromorphic at p. Q.E.D.

Note that if in 3.5.1 we require that each $h_{j}$ map $A_{j}$ into itself for all $j$, then $h^{*}$ maps $A$ into itself. In fact, when $a=\left(a_{1}, \ldots, a_{n}\right) \in A_{1} \oplus \cdots \oplus A_{n}, h^{*}(a)=\left(h_{1}\left(a_{1}\right)\right.$, $\left.\ldots, h_{n}\left(a_{n}\right)\right)$.

LEMMA 3.5.2. Let $A$ and $A^{\prime}$ be commutative complex Banach algebras with identity. Suppose that $f: A \rightarrow A^{\prime}$ and $f^{\prime}: A^{\prime} \rightarrow A$ are continuous algebra with identity homomorphisms so that $f^{\prime} \circ f=1_{A}$ and $f \circ f^{\prime}=1_{A^{\prime}}$. Let $h$ be meromorphic on $A_{\infty}$, define $h^{\prime}: A_{\infty}^{\prime} \rightarrow A_{\infty}^{\prime}$ by $h^{\prime}=f_{*} \circ h \circ f_{*}^{\prime}$. Then $h^{\prime}$ is meromorphic on $A_{\infty}^{\prime}$. Furthermore, if $h$ maps $A$ into itself, then $h^{\prime}$ maps $A^{\prime}$ into itself.

Proof. Let $p^{\prime} \in A_{\infty}^{\prime}$, set $p=f_{*}^{\prime}\left(p^{\prime}\right)$, and choose $X_{1}$ and $X_{2}$ from $G(A)$ so that $X_{1}(p)=0=X_{2}(h(p))$. Choose $\delta>0$ so that $X_{2} \circ h \circ X_{1}^{-1}$. maps $B(0 ; \delta)$ into $A$; $X_{2} \circ h \circ X_{1}^{-1}$ is thus holomorphic on $B(0 ; \delta)$ with power series expansion $\sum c_{n} a^{n}$. Set

$$
X_{1}^{\prime}=f^{*}\left(X_{1}\right), \quad X_{2}^{\prime}=f^{*}\left(X_{2}\right), \quad \text { and } \quad U^{\prime}=X_{1}^{\prime-1}\left(B\left(0 ; \delta /\left\|f^{\prime}\right\|\right)\right)
$$

Since

$$
X_{1}^{\prime}\left(p^{\prime}\right)=f^{*}\left(X_{1}\right) \circ f_{*} \circ f_{*}^{\prime}\left(p^{\prime}\right)=\left(\text { by 1.4.7) } f_{*} \circ X_{1}(p)=0\right.
$$


$U^{\prime}$ is an open connected neighborhood of $p$; clearly $X_{1}^{\prime}$ maps $U^{\prime}$ into $A^{\prime}$. Furthermore when $b^{\prime} \in A^{\prime}$ and $\left\|b^{\prime}\right\|<\delta /\left\|f^{\prime}\right\|$, then

$$
\begin{aligned}
X_{2}^{\prime} \circ h^{\prime} \circ X_{1}^{\prime-1}\left(b^{\prime}\right) & =X_{2}^{\prime} \circ h^{\prime} \circ f^{*}\left(X_{1}^{-1}\right) \circ f_{*} \circ f_{*}^{\prime}\left(b^{\prime}\right) \\
& =X_{2}^{\prime} \circ h^{\prime} \circ f_{*} \circ X_{1}^{-1} \circ f^{\prime}\left(b^{\prime}\right)=f^{*}\left(X_{2}\right) \circ f_{*} \circ h \circ f_{*}^{\prime} \circ f_{*} \circ X_{1}^{-1} \circ f^{\prime}\left(b^{\prime}\right) \\
& =f_{*} \circ X_{2} \circ h \circ X_{1}^{-1} \circ f^{\prime}\left(b^{\prime}\right)=f_{*}\left(\sum c_{n}\left(f^{\prime}\left(b^{\prime}\right)\right)^{n}\right)=\sum f\left(c_{n}\right)\left(b^{\prime}\right)^{n} .
\end{aligned}
$$

Thus $X_{2}^{\prime} \circ h^{\prime} \circ X_{1}^{\prime-1}$ is represented by a convergent power series on $B\left(0 ; \delta /\left\|f^{\prime}\right\|\right)$ $=X_{1}^{\prime}\left(U^{\prime}\right)$. Therefore $h^{\prime}$ is meromorphic at $p^{\prime}$.

Furthermore, if $h(A) \subseteq A$ and $b^{\prime} \in A^{\prime}$, then $h^{\prime}\left(b^{\prime}\right)=f_{*} \circ h \circ f_{*}^{\prime}\left(b^{\prime}\right)=f\left(h\left(f^{\prime}\left(b^{\prime}\right)\right)\right) \in A^{\prime}$. Q.E.D.

We can now define the concept of "algebraic polynomial in $A$ ". By a partition of the identity for $A$ we mean a finite sequence $j_{1}, \ldots, j_{n}$ of distinct nonzero idempotents so that

$$
j_{k} j_{i}=0 \quad \text { when } k \neq i \text {, and } \sum_{k=1}^{n} j_{k}=1
$$

Let $j_{i}, \ldots, j_{n}$ be a partition of the identity in $A$. For $1 \leqq i \leqq n$ let $A_{i}$ be the commutative Banach algebra with identity obtained by renorming the closed subalgebra $j_{i} A$ via its regular representation; thus if \|\|$\|$ denotes the norm in $A_{i}$, \|\|$\|$ is equivalent to the norm in $A_{i}$ induced by $A$, and $\left\|j_{i}\right\|=1$. Let $q_{1}: A_{1} \oplus \cdots \oplus A_{n}$ $\rightarrow A$ and $q_{2}: A \rightarrow A_{1} \oplus \cdots \oplus A_{n}$ be defined by

$$
q_{1}\left(a_{1}, \ldots, a_{n}\right)=a_{1}+\cdots+a_{n}, \quad q_{2}(a)=\left(j_{1} a, \ldots, j_{n} a\right) .
$$

It is not hard to see that $q_{1}$ and $q_{2}$ are continuous algebra with identity homomorphisms so that $q_{2}$ is the inverse mapping of $q_{1}$.

A function $\xi: A_{\infty} \rightarrow A_{\infty}$ is an algebraic polynomial (al.p.) on $A$ iff there are $j_{1}, \ldots, j_{n}$ and $h_{1}, \ldots, h_{n}$ so that

(1) $j_{1}, \ldots, j_{n}$ is a partition of the identity in $A$,

(2) if for each $i, 1 \leqq i \leqq n$, we let $A_{i}$ be the commutative $B$-algebra with identity obtained by renorming $j_{i} A$ via the regular representation. Then each $h_{i}$ is a mapping of $A_{i \infty}$ into itself which is either an $A_{i}$ simple algebraic polynomial or an $A$-valued constant mapping, and

(3) if $h^{*}$ is the meromorphic function on $\left(A_{1} \oplus \cdots \oplus A_{n}\right)_{\infty}$ constructed from $h_{1}, \ldots, h_{n}$ as in 3.5.1, and $q_{1}$ and $q_{2}$ are the isomorphisms of $A_{1} \oplus \cdots \oplus A_{n}$ and $A$ introduced above, then $\xi=q_{1} \circ \circ h^{*} \circ q_{2^{*}}$.

3.5.2 and the note preceding 3.5.2 show that each algebraic polynomial is a meromorphic function defined on $A_{\infty}$ which maps $A$ into $A$. Thus the restriction to $A$ of an algebraic polynomial is an analytic polynomial.

THEOREM 3.5.3. If $g$ is an analytic polynomial, then $g$ is the restriction to $A$ of an algebraic polynomial. 
Proof. Let $p_{0}=\lim _{z \leqq} g(z)$, and let $\sum c_{n} a^{n}$ be the power series expansion of $g$ on $A$. If $F$ is a maximal ideal,

$$
F_{*}\left(p_{0}\right)=F_{*}\left(\lim _{z \rightarrow \infty} g(z)\right)=\lim _{z \rightarrow \infty} g_{F}(z) .
$$

Since $\lim _{z \rightarrow \infty} g_{F}(z)$ exists, $g_{F}$ cannot have an essential singularity at $\infty$. For each maximal ideal $F$, set $n(F)=0$ if $g_{F}$ is constant, and set $n(F)=$ the degree of $g_{F}$ if $g_{F}$ is a nonconstant polynomial. We will show that $n(F)$ is continuous on $\mathscr{M}$, i.e., $n(F)$ is constant in a neighborhood of each maximal ideal. Fix a maximal ideal $F^{\prime}$ of $A$.

If $n\left(F^{\prime}\right)=0$, then $F_{*}^{\prime}\left(p_{0}\right)=\lim _{z \rightarrow \infty} g_{F^{\prime}}(z)$ is finite. By the continuity of $\hat{p}_{0}$, choose a neighborhood $V^{\prime}$ of $F^{\prime}$ so that

$$
\lim _{z \rightarrow \infty} g_{F}(z)=F_{*}\left(p_{0}\right)
$$

is finite when $F$ is in $V_{1}$. Thus $n(F)=0$ on $V_{1}$.

Now suppose $n\left(F^{\prime}\right)>0$. Then

$$
F_{*}^{\prime}\left(p_{0}\right)=\lim _{z \rightarrow \infty} g_{F^{\prime}}(z)=\infty
$$

By 2.4.2 choose a neighborhood $U$ of $p_{0}$ in $A$, and a neighborhood $N_{0}$ of $F^{\prime}$ in $\mathscr{M}$ so that either $\hat{p}(F)=\infty$ or $\hat{p}(F)$ is finite and $|\hat{p}(F)| \geqq 1$ when $p$ is in $U$ and $F$ is in $N_{0}$. Since $\lim _{z \rightarrow \infty} g(z)=p_{0}$, choose $s>0$ so that $g(z) \in U$ when $|z| \geqq s$. Thus $\left|g_{F}(z)\right|$ $=|F(g(z))| \geqq 1$, if $|z| \geqq s$ and $F \in N_{0}$. By the continuity of $F \rightarrow g_{F}$ choose a neighborhood $N_{1}$ of $F^{\prime}$ so that $N_{1} \subseteq N_{0}$ and

$$
\left|g_{F}(z)-g_{F^{\prime}}(z)\right| \leqq \frac{1}{2} \quad \text { when } F \in N_{1} \text { and }|z|=s .
$$

For $F$ in $N_{1}$ it follows from Rouche's theorem that $g_{F}$ and $g_{F}$ have the same number of zeros on $K(0 ; s)$; thus $g_{F}$ and $g_{F}$ have the same number of zeros on $C$, i.e. $n\left(F^{\prime}\right)$ zeros. So for $F$ in $N_{1}, g_{F}$ is a polynomial of degree $n\left(F^{\prime}\right) ; n(F)=n\left(F^{\prime}\right)$. Thus $n(F)$ is continuous on $\mathscr{M}$.

For each nonnegative integer $n$, set $\mathscr{M}_{n}=\{F: n(F)=n\}$. Each $\mathscr{M}_{n}$ is open and closed in $\mathscr{M}$, and $\mathscr{M}$ is the disjoint union of all the $\mathscr{M}_{n}$. Since $\mathscr{M}$ is compact, there is some $N>0$ so that $\mathscr{M}_{N}$ is nonempty, and $\mathscr{M}_{n}$ is empty for $n>N$. For $0 \leqq n \leqq N$ let $j_{n}^{\prime}$ be the idempotent of $A$ so that (Rickart $[9$, p. 168])

$$
\left(j_{n}^{\prime \wedge}\right)^{-1}(1)=\mathscr{M}_{n} \text {. }
$$

Let $j_{0}, \ldots, j_{M}$ be the sequence obtained from $j_{0}^{\prime}, \ldots, j_{N}^{\prime}$ by omitting all the zero idempotents; $j_{0}, \ldots, j_{M}$ is a partition of the identity and $j_{M}=j_{N}^{\prime}$. For $0 \leqq i \leqq M$ let $A_{i}$ be the commutative Banach algebra with identity obtained by renorming $j_{i} A$ via the regular representation; let $P_{i}^{\prime}: A \rightarrow A_{i}$ be the continuous homomorphism defined by $P_{i}^{\prime}(a)=j_{i} a$. Define $g_{i}: A_{i} \rightarrow A_{i}$ by

$$
g_{i}\left(a_{i}\right)=\sum_{n=0}^{\infty} j_{i} c_{n}\left(a_{i}\right)^{n}
$$


Then

$$
\begin{aligned}
p_{0 i} & =P_{i \star}^{\prime}\left(p_{0}\right)=P_{i \bullet}^{\prime}\left(\lim _{z \rightarrow \infty} g(z)\right) \\
& =\lim _{z \rightarrow \infty} P_{i}^{\prime}(g(z))=\lim _{z \rightarrow \infty} g_{i}(z) .
\end{aligned}
$$

Thus $g_{i}$ is an analytic polynomial on $A_{i}$. For $0 \leqq i \leqq M$ let $u(i)$ be the integer satisfying $j_{u(i)}^{\prime}=j_{i}$.

Suppose $u(i)>0$. Let $G_{i}$ be a maximal ideal of $A_{i}$, set $F_{i}=$ the maximal ideal of $A$ given by $G_{i} \circ P_{i}^{\prime}$. If $a \in A, F_{i}(a)=G_{i}\left(j_{i} a\right)$. Since $F_{i}\left(j_{u(i)}^{\prime}\right)=G_{i}\left(j_{i}\right)=1$, $n\left(F_{i}\right)=u(i)$. So if $n>u(i), \quad G_{i}\left(j_{i} c_{n}\right)=F_{i}\left(c_{n}\right)=0$, while $G_{i}\left(j_{i} c_{u(i)}\right)=F_{i}\left(c_{u(i)}\right) \neq 0$. Therefore $g_{i}$ is a simple analytic polynomial on $A_{i}$. By 3.3 .4 let $h_{i}$ be the simple algebraic polynomial on $A_{i}$ whose restriction to $A_{i}$ is $g_{i}$.

Suppose $u(i)=0$. By the above argument, we see that if $n>0$, then $G_{i}\left(j_{i} c_{n}\right)=0$. Therefore

$$
G_{i \bullet}\left(p_{0 i}\right)=\lim _{z \rightarrow \infty} G_{i}\left(g_{i}(z)\right)=G_{i}\left(j_{i} c_{0}\right) \in C .
$$

Thus $\infty$ is not in the spectrum of $p_{0 i}$ in $A_{i \infty}$, so $p_{0 i}$ lies in $A_{i}$. By Liouville's theorem $g_{i}$ is identically constant on $A_{i}$. Let $h_{i}$ be the constant function on $A_{i \infty}$ whose restriction to $A_{i}$ is $g_{i}$.

Use the $h_{0}, \ldots, h_{M}$ to construct the meromorphic function $h^{*}$ on $\left(A_{0} \oplus \cdots \oplus A_{M}\right)_{\infty}$ via 3.5.1. Define the algebraic polynomial $\xi: A_{\infty} \rightarrow A_{\infty}$ via $\xi=q_{1} \circ \circ h^{*} \circ q_{2^{\circ}}$, where $q_{1}$ and $q_{2}$ are defined as on $p$ via $j_{0}, \ldots, j_{M}$. If $a \in A$, then

$$
\begin{aligned}
\xi(a) & =q_{1}\left(h_{0}\left(j_{0} a\right), \ldots, h_{M}\left(j_{M} a\right)\right) \\
& =q_{1}\left(\sum_{n}\left(j_{0} c_{n}\right)\left(j_{0} a\right)^{n}, \ldots, \sum_{n}\left(j_{M} c_{n}\right)\left(j_{M} a\right)^{n}\right) \\
& =\sum_{i=0}^{M} \sum_{n=0}^{\infty}\left(j_{i} c_{n}\right)\left(j_{i} a\right)^{n}=\sum_{i=0}^{M} j_{i} \sum_{n=0}^{\infty} c_{n} a^{n}=g(a) .
\end{aligned}
$$

Thus $g$ is the restriction to $A$ of the generalized polynomial $\xi$. Q.E.D.

Note that the $j_{n}^{\prime}$ of the preceding proof can be constructed directly; i.e. without invoking the Silov theorem that for any clopen subset $\mathscr{M}_{1}$ of $\mathscr{M}$ there is an idempotent $j$ in $A$ so that $\hat{j}^{-1}(1)=\mathscr{M}_{1}$. (The details of the construction appear in the author's dissertation.) Since the proof of the Silov theorem depends on the theory of functions of several complex variables, we thus avoid having a portion of the theory of analytic functions of one algebra variable depend on the theory of analytic functions of several complex variables.

We now present some illustrative examples of the concepts of simple analytic polynomial and analytic polynomial.

EXAMPLE 3.5.4. Suppose $r$ is a nonnilpotent element of the radical, define $g: A \rightarrow A$ by $g(a)=a(1-r a)^{-1} \cdot g$ is a simple analytic polynomial, but $g$ is not a polynomial. 
Example 3.5.5. Let $A=C[0,1]$, define $x_{1}:[0,1] \rightarrow C$ by $x_{1}(t)=t$, define $g: A \rightarrow A$ by $g(a)=x_{1} a$. $g$ is a polynomial but $g$ is neither a simple analytic polynomial nor an analytic polynomial.

EXAmple 3.5.6. Let $A=C \times C$, with pointwise operations and the sup norm. Set $x_{1}=(1,0)$ and $x_{0}=(0,1)$, define $g: A \rightarrow A$ by $g(a)=x_{0}+x_{1} a . g$ is an analytic polynomial, but $g$ is not a simple analytic polynomial.

EXAMPLE 3.5.7. Let $A$ be the algebra of bounded sequences of complex numbers, with pointwise operations and the sup norm. For $n \geqq 0$ set $x_{n}=\left(\delta_{n k} / k !\right)_{k=0}^{\infty}$, where $\delta_{n k}$ is the Kronecker $\delta$. Define $g: A \rightarrow A$ by $g(a)=\sum x_{n} a^{n}$. It follows from 3.5.3 that $g$ is not an analytic polynomial.

\section{BIBLIOGRAPHY}

1. E. K. Blum, A theory of analytic functions in Banach algebras, Trans. Amer. Math. Soc. 78 (1955), 343-370.

2. N. G. de Bruijn, Function theory in Banach algebras, Ann. Acad. Sci. Fenn. Ser. A 250/5 (1958).

3. - Verallgemeinerte Riemannsche Sphären, Nachr. Akad. Wiss. Göttingen Math.Phys. Kl. 2 (1959), 279-292.

4. B. Glickfeld, Contributions to the theory of holomorphic functions in commutative Banach algebras with identity, Dissertation, Columbia Univ., New York, 1964.

5. E. Hille and R. S. Phillips, Functional analysis and semi-groups, Amer. Math. Soc. Colloq. Publ., Vol. 31, rev. ed., Amer. Math. Soc., Providence, R. I., 1957.

6. E. R. Lorch, The theory of analytic functions in normed abelian vector rings, Trans. Amer. Math. Soc. 54 (1943), 414-425.

7. - - The structure of normed abelian rings, Bull. Amer. Math. Soc. 50 (1944), 447-463.

8. - Normed rings-the first decade, Proc. symposium on spectral theory and differential problems, Oklahoma A \& M, Stillwater, Okla., 1955, pp. 249-258.

9. C. Rickart, Banach algebras, Van Nostrand, New York, 1960.

10. S. Saks and A. Zygmund, Analytic functions, Hafner, New York, 1952.

11. A. E. Taylor, Spectral theory of closed distributive operators, Acta Math. 84 (1951), 189-224.

HARVARD University,

CAmbridge, Massachusetts 